\title{
Synthesis of Nerol Derivatives Containing a 1,2,3-Triazole Moiety and Evaluation of Their Activities against Cancer Cell Lines
}

\author{
Róbson R. Teixeira, ${ }^{\oplus *}{ }^{* a}$ Adalberto M. da Silva, ${ }^{b}$ Raoni P. Siqueira, ${ }^{c}$ Victor Hugo S. Gonçalves, ${ }^{c}$ \\ Higor S. Pereira, ${ }^{c}$ Rafaela S. Ferreira, ${ }^{d}$ Adilson V. Costa, ${ }^{e}$ Eduardo B. de Melo, ${ }^{f}$ Fávero R. Paula, ${ }^{g}$ \\ Márcia M. C. Ferreira ${ }^{\circledR h}{ }^{h}$ and Gustavo C. Bressan ${ }^{c}$
}

${ }^{a}$ Departamento de Química, Universidade Federal de Viçosa, Av. P.H. Rolfs, S/N, 36570-900 Viçosa-MG, Brazil

${ }^{b}$ Instituto Federal de Educação, Ciência e Tecnologia Catarinense, Campus Araquari, Rodovia BR 280, km 27, CP 21, 89245-000 Araquari-SC, Brazil

'Departamento de Bioquímica e Biologia Molecular, Universidade Federal de Viçosa, Av. P.H. Rolfs, S/N, 36570-900 Viçosa-MG, Brazil

${ }^{d}$ Departamento de Bioquímica e Imunologia, Universidade Federal de Minas Gerais, Av. Antônio Carlos 6627, 31270-901 Belo Horizonte-MG, Brazil

eDepartamento de Química e Física, Universidade Federal do Espírito Santo, Alto Universitário, S/N, Guararema, 29500-000 Alegre-ES, Brazil

${ }^{f}$ Laboratório de Química Medicinal e Ambiental Teórica (LQMAT), Departamento de Farmácia, Universidade Estadual do Oeste do Paraná (UNIOESTE), Rua Universitária 2069, 85819-110 Cascavel-PR, Brazil

${ }^{8}$ Departamento de Farmácia, Universidade Federal do Pampa (UNIPAMPA), BR 472, km 592, P.O. Box 118, 97500-970 Uruguaiana-RS, Brazil

hInstituto de Química, Universidade Estadual de Campinas, 13084-971 Campinas-SP, Brazil

In the present investigation, a collection of twenty two nerol derivatives, containing 1,2,3-triazolic appendages, was synthesized and screened in vitro for their cytotoxic activity against HL60, Nalm6, and Jurkat human leukemia cells as well as against B16F10 (melanoma cell line). In most cases, derivatives were able to reduce cell viability. The most potent compound (Z)-4-(((3,7-dimethylocta-2,6-dien-1-yl)oxy)methyl)-1-(4-(trifluoromethoxy)benzyl)$1 H-1,2,3$ triazole showed antiproliferative activity against Jurkat cells and reduced B16F10 cell migration. Physicochemical properties of the compounds were calculated in order to evaluate their potential for drug development. Most of the evaluated physicochemical parameters seemed to be favorable for drug development. In addition, for a better understanding of the biological activity results, 3D quantitative structure-activity relationship (QSAR) studies were carried out. 3D-QSAR studies indicate that the anticancer activities observed for the cell lines HL60 and Jurkat may occur by a similar mechanism of action and the same was found for the Nalm6 and B16F10 cell lines.

Keywords: nerol, 3D-QSAR, leukemia, melanoma, click chemistry

\section{Introduction}

Mother Nature is a formidable reservoir of compounds that can be explored for the search and development of pharmaceuticals. ${ }^{1-6}$ Several drugs have been discovered via

*e-mail: robsonr.teixeira@ufv.br screening of natural products from plants, animals, marine organisms and microorganisms. In the particular case of cancer, from 1/1/1981 to 12/31/2014, Newman an Cragg ${ }^{7}$ pointed out that among 174 new chemical entities approved as anticancer drugs, $136(78 \%)$ corresponded to small molecules. Moreover, 113 out of 136 (83\%) were either natural products per se or based on them, or mimicked 
natural products in one form or another. ${ }^{7}$ Vincristine, irinotecan, etoposide and paclitaxel are examples of natural product based drugs that have been used in cancer chemotherapy. ${ }^{8}$ It should be mentioned that compounds from natural sources have been optimized through evolution process and present physicochemical properties well-suited for successful drug development into the market.

Essential oils can be defined as odorous and volatile mixtures found in $10 \%$ of the plant kingdom and stored in special delicate secretory structures. They are composed of very complex natural mixtures containing hydrocarbons (mainly terpenoids) and oxygenated compounds (alcohols, esters, ethers, aldehydes, ketones, lactones, phenols and phenol ethers). Essential oils are widely used in medicine, in perfumes, cosmetics and bath products, as flavoring agents in food and drink, and in many other manufacturing areas. Since immemorial times, their therapeutic value has been recognized by man. ${ }^{9}$ These oils are a valuable source of very interesting and powerful natural plant products.

One very common compound found in essential oil is nerol [(2Z)-3,7-dimethylocta-2,6-dien-1-ol]. From the chemical standpoint, nerol (Figure 1) is a monoterpene commonly used in fragrances, soaps, shampoos, cleaners and detergents. Moreover, this compound is found in several medicinal plants such as Lippia spp. and Melissa officinalis L. Investigations have ascribed antimicrobial, antioxidant, antiviral and anxiolytic activities to these plant species which, in turn, have been attributed to the presence of nerol. ${ }^{10}$

Yamamoto et al. ${ }^{11}$ investigated the effect of phosphatidylated terpenes alcohols on human prostate PC-3 and human leukemia HL60 cell lines. Treatment of these cell lines with phosphatidyl-nerol derivative (Figure 1) at $100 \mu \mathrm{mol} \mathrm{L}^{-1}$ and after $72 \mathrm{~h}$ led to a decrease of $70 \%$ in cell viability. Nerol itself had no effect on cell line viabilities even at $400 \mu \mathrm{mol} \mathrm{L}^{-1} .^{11}$

Nowadays, several chemotherapeutic regimes have been successfully implemented for fighting cancer. However, most treatments are associated with severe side-effects and resistance, highlighting the necessity of a constant effort toward the development of novel therapies..$^{12-16}$

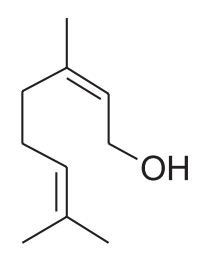

Nerol

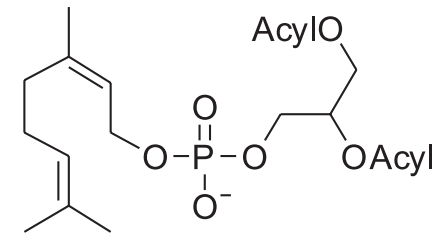

Phosphatidyl-nerol

Figure 1. Structures of nerol and nerol derivatives.

In the discovery of new chemotherapeutic agents, one important aspect is the use of synthetic strategies that allow the rapid construction of compounds or libraries of compounds during the process of drug discovery or lead optimization. The very fast preparation of groups of bioactive compounds speeds up the process of drug discovery and lead optimization. The click chemistry has emerged as one powerful tool in drug discovery. ${ }^{17-19}$ As defined by Sharpless and co-workers, ${ }^{20}$ the click chemistry refers to reactions that are regiospecific in nature and can be performed easily, yielding highly pure compounds with fewer efforts. The most popular reaction that meet the aforementioned criteria is the copper(I)-catalyzed azide-alkyne cycloaddition (CuAAC reaction). In this transformation, a 1,2,3-triazole ring 1,4-disubstituted is obtained from a terminal alkyne and an azide and it is commonly known in the literature as click reaction. A variety of bioactive compounds, ${ }^{21}$ including anticancer agents, ${ }^{22-27}$ have been synthesized using the click reaction as the key step. It should be mentioned that 1,2,3-triazole ring is an attractive unit, since it is stable to metabolic degradation, oxidative/reducing conditions, and improves solubility by actively binding to biomolecular targets. ${ }^{28}$ This ring is also known as the pharmacophoric group. ${ }^{21}$

Considering the cytotoxicity activity of nerol, the necessity to develop new chemotherapeutic agents for cancer treatment, and the anticancer activity related to 1,2,3-triazole derivatives, we herein described the structural modification of nerol into derivatives containing 1,2,3-triazole portions (Figure 1).

The key reaction involved in the preparation of twenty two nerol analogues corresponded to the click reaction between several benzyl azides and alkylated nerol derivatives. The cytotoxicity of the synthesized compounds was evaluated against human leukemias and murine melanoma cell lines. Physicochemical properties were also calculated in order to evaluate the potential of the synthesized nerol derivatives for drug development. 3D quantitative structure-activity relationship (QSAR) studies were carried out in order to have a better understanding about the anticancer mechanism against each cell line.

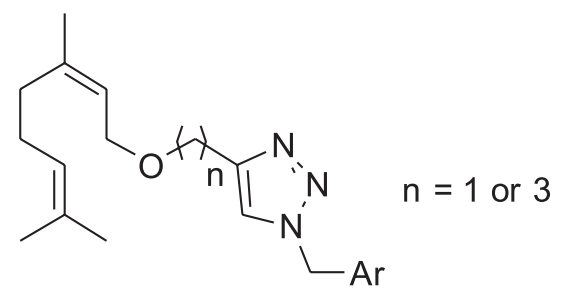

General structure of compounds herein investigated 


\section{Experimental}

\section{Generalities}

Solvents were purchased from Vetec (Rio de Janeiro, Brazil). Nerol, benzyl alcohols, pent-4-yn-1-ol, methanesulfonyl chloride, sodium azide, triethylamine and propargyl bromide solution (80 wt.\% in toluene, containing $0.3 \%$ of magnesium as stabilizer) were procured from Sigma-Aldrich (St. Louis, MO, USA) and used as received. ${ }^{1} \mathrm{H}$ and ${ }^{13} \mathrm{C}$ nuclear magnetic resonance (NMR) spectra were recorded on a Varian Mercury 300 instrument (Varian, Palo Alto, CA, USA) at 300 and $75 \mathrm{MHz}$, respectively, using $\mathrm{CDCl}_{3}$ as solvent. ${ }^{1} \mathrm{H}$ NMR data are presented as follows: chemical shift $(\delta)$ in ppm, multiplicity, number of protons, $J$ values in hertz $(\mathrm{Hz})$. Multiplicities are shown as the following abbreviations: s (singlet), brs (broad singlet), $\mathrm{d}$ (doublet), dd (double of a doublet), $\mathrm{t}$ (triplet), dt (double of a triplet), q (quartet), quint (quintet), m (multiplet). For fluorine containing derivatives, the multiplicity of some carbon signals are described along with $J$ values in hertz. Infrared (IR) spectra were obtained using a Varian 660-IR equipped with GladiATR (Varian, Palo Alto, CA, USA) scanning from 4000 to $500 \mathrm{~cm}^{-1}$. Analytical thin layer chromatography (TLC) analyses were conducted on aluminum backed precoated silica gel plates using different solvent systems and were visualized using potassium permanganate solution, phosphomolybdic acid solution and/or UV light. Column chromatography was performed using silica gel (60-230 mesh).

\section{Synthesis}

\section{Synthesis of benzyl methanesulfonate (2a)}

Benzyl alcohol (1.08 g, $10.0 \mathrm{mmol})$ and anhydrous dichloromethane (DCM, $20 \mathrm{~mL}$ ) were added to a $50 \mathrm{~mL}$ round-bottom flask under nitrogen atmosphere. The mixture was cooled to $-50{ }^{\circ} \mathrm{C}$ and then triethylamine $(2.78 \mathrm{~mL}$, $20.0 \mathrm{mmol}$ ) was added, followed by slow addition of methanesulfonyl chloride (Ms-Cl, $1.20 \mathrm{~mL}, 15.0 \mathrm{mmol}$ ). The progress of the reaction was monitored by TLC. After its completion, $20 \mathrm{~mL}$ of water were added. The layers were separated and the organic layer was washed with $1 \% \mathrm{~m} / \mathrm{v} \mathrm{HCl}$ aqueous solution $(3 \times 15 \mathrm{~mL})$, followed by $\mathrm{NaHCO}_{3}$ saturated aqueous solution $(3 \times 5 \mathrm{~mL})$, dried over $\mathrm{MgSO}_{4}$, and concentrated under reduced pressure. The compound 2a was obtained in $87 \%$ yield $(1.63 \mathrm{~g}, 8.76 \mathrm{mmol})$ and it was not submitted to any further purification procedure.

Ester sulfonates $\mathbf{2 b}$-2k (Scheme 1) were synthesized using this same methodology. The spectroscopic data of these compounds were in agreement with those reported in the literature. ${ }^{29}$

\section{Synthesis of azidomethylbenzene (3a)}

Benzyl methanesulfonate (2a) (930 mg, $5.00 \mathrm{mmol}$ ) was added to a $50 \mathrm{~mL}$ round-bottom flask containing $10 \mathrm{~mL}$ of DMSO (dimethyl sulfoxide) and $1.33 \mathrm{~g}$ (20.0 mmol) of sodium azide. The reaction mixture was stirred at room temperature for $6 \mathrm{~h}$. After that, $10 \mathrm{~mL}$ of water $\left(5^{\circ} \mathrm{C}\right)$ were added to the reaction mixture and the resulting aqueous phase was extracted with ethyl acetate $(3 \times 15 \mathrm{~mL})$. The organic extracts were combined and the resulting organic phase was washed with saturated $\mathrm{NaCl}$ aqueous solution $(3 \times 15 \mathrm{~mL})$, dried over $\mathrm{MgSO}_{4}$, filtered and concentrated under reduced pressure. Compound 3a was obtained in $97 \%$ yield (650 mg, $4.88 \mathrm{mmol}$ ) and it was not submitted to any further purification procedure.

Benzyl azides 3b-3k (Scheme 1) were synthesized using this same methodology. The spectroscopic data of these compounds are in agreement with those reported in the literature. ${ }^{29}$

Synthesis of (Z)-3,7-dimethyl-1-(prop-2-yn-1-yloxy)octa2,6-diene (7)

A $125 \mathrm{~mL}$ round bottom flask, under nitrogen atmosphere, was charged with sodium hydride (480 mg, $20.0 \mathrm{mmol}$ ) and $10 \mathrm{~mL}$ of anhydrous THF (tetrahydrofuran). The resulting mixture was heated to $40{ }^{\circ} \mathrm{C}$. Then, (2Z)-3,7-dimethylocta-2,6-dien-1-ol (nerol, compound 6 in Scheme 1), dissolved in $2 \mathrm{~mL}$ of anhydrous THF was added slowly. The system was kept at $40{ }^{\circ} \mathrm{C}$ and magnetically stirred for $15 \mathrm{~h}$. After that, a solution of propargyl bromide (1.33 mL, $15.0 \mathrm{mmol})$ in THF (2 mL) was slowly added. The reaction mixture was maintained at $40{ }^{\circ} \mathrm{C}$ and magnetically stirred for additional $7 \mathrm{~h}$. Upon completion of the reaction as noticed by TLC analysis, $20 \mathrm{~mL}$ of $2 \mathrm{~mol} \mathrm{~L}^{-1}$ $\mathrm{HCl}$ aqueous solution were added. The aqueous layer was extracted with ethyl acetate $(3 \times 20 \mathrm{~mL})$. The organic phases were combined and the resulting organic layer was washed with water $(20 \mathrm{~mL})$ and brine $(20 \mathrm{~mL})$, dried over $\mathrm{MgSO}_{4}$, filtered, and concentrated under reduced pressure. The crude reaction product was purified by silica gel column chromatography using a solvent mixture of hexane/ethyl acetate $(10: 1 \mathrm{v} / \mathrm{v})$. Compound 7 was obtained as brown oil in $97 \%$ yield $(1.88 \mathrm{~g}, 9.70 \mathrm{mmol})$. TLC: $\mathrm{Rf}=0.50$ (hexane/ ethyl acetate 10:1 v/v); IR (ATR) $\bar{v}_{\max } / \mathrm{cm}^{-1} 3303,2965$, 2926, 2855, 2114, 1666, 1591, 1444, 1376, 1251, 1070, 932, 828, 664, 628; ${ }^{1} \mathrm{H}$ NMR (300 MHz, $\left.\mathrm{CDCl}_{3}\right) \delta 1.60$ (s, $3 \mathrm{H}), 1.68$ (s, 3H), 1.75 (s, 3H), 2.09 (s, 4H), 2.40 (t, 1H, $J 2.3 \mathrm{~Hz}), 4.05$ (d, 2H, J 7.0 Hz), 4.11 (d, 2H, J $2.3 \mathrm{~Hz}$ ), 5.09 (brs, 1H), 5.33 (t, 1H, J 7.0 Hz); ${ }^{13} \mathrm{C} \mathrm{NMR} \mathrm{(75} \mathrm{MHz,}$ 


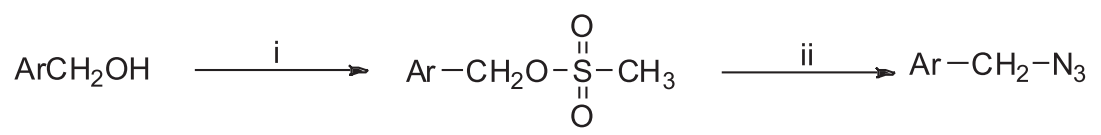

(1)<smiles>C#CCCCO</smiles>

(4)<smiles>C#CCOC/C=C(/C)CCC=C(C)C</smiles>

(7)<smiles>CC(C)=CCCC(C)=CCOCc1cn(CBr)nn1</smiles>

$(9 \mathrm{a}-9 \mathrm{k})$

$A r=$<smiles>CC(C)(C)c1ccc(F)cc1</smiles><smiles>CC(C)(C)c1ccc(Cl)cc1</smiles><smiles>CC(C)(C)c1ccc(Br)cc1</smiles><smiles>CC(C)(C)c1ccc(I)cc1</smiles>

(d)

(a) (b)

(c)

(e)
(2)

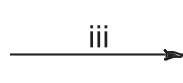<smiles>CCC(=CCCO)CCC=C(C)C</smiles>

(6)<smiles>C#CCCCOS(C)(=O)=O</smiles>

(5)

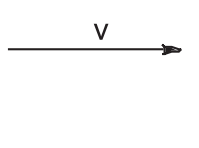<smiles>C#CCCCOCC=C(C)CCC=C(C)C</smiles>

(8)<smiles>CC(C)=CCCC(C)=CCOCCCc1cn(C[Al])nn1</smiles>

(10a-10k)

(f)

(g)

(h)

(i)<smiles>Cc1ccc(F)c(F)c1</smiles>

(j)<smiles>CC(C)(C)c1cc(Cl)ccc1Cl</smiles>

(k)

Scheme 1. Reagents and conditions: (i) and (iii) TEA, Ms-Cl, DCM, $-50{ }^{\circ} \mathrm{C}$; (ii) $\mathrm{NaN}_{3}$, DMSO, r.t.; (iv) NaH, THF, propargyl bromide, $40{ }^{\circ} \mathrm{C}$; (v) $\mathrm{NaH}$, THF, compound $5,40{ }^{\circ} \mathrm{C}$; (vi) and (vii) sodium ascorbate, $\mathrm{CuSO}_{4} \cdot 5 \mathrm{H}_{2} \mathrm{O}, \mathrm{CH}_{2} \mathrm{Cl}_{2} / \mathrm{H}_{2} \mathrm{O}$.

$\left.\mathrm{CDCl}_{3}\right) \delta 17.6,23.5,25.6,26.7,32.2,56.7,65.7,74.1,80.0$ 120.9, 123.8, 132.0, 141.7; mass spectrometry-electron ionization (MS-EI), $\mathrm{m} / \mathrm{z}(\%): 192(3) \mathrm{C}_{13} \mathrm{H}_{20} \mathrm{O}\left[\mathrm{M}^{+*}\right], 177$ (2), 153 (2), 149 (3), 136 (6), 121 (25), 107 (13), 93 (60), 80 (31), 69 (100), 55 (16), 41 (81).

\section{Synthesis of pent-4-yn-1-yl methanesulfonate (5)}

Pent-4-yn-1-ol (1.68 g, $20.0 \mathrm{mmol})$ and dichloromethane $(20 \mathrm{~mL})$ were added to a $50 \mathrm{~mL}$ round-bottom flask under nitrogen atmosphere. The mixture was cooled to $-50{ }^{\circ} \mathrm{C}$ and triethylamine $(5.60 \mathrm{~mL}, 40.0 \mathrm{mmol})$ was added. After that, methanesulfonyl chloride was added slowly $(2.32 \mathrm{~mL}$, $30.0 \mathrm{mmol}$ ) to the reaction mixture under continuous stirring. The progress of the reaction was monitored by TLC. After completion of it, $10 \mathrm{~mL}$ of water were added. The organic phase was washed with $1 \% \mathrm{HCl}$ aqueous solution $(3 \times 15 \mathrm{~mL})$ followed by saturated $\mathrm{NaHCO}_{3}$ aqueous solution $(3 \times 5 \mathrm{~mL})$, dried over $\mathrm{MgSO}_{4}$, and concentrated under reduced pressure. Compound $\mathbf{5}$ was obtained as yellow oil in $92 \%$ yield $(3.00 \mathrm{~g}, 18.0 \mathrm{mmol})$ and 
it was not submitted to any further purification procedure. TLC: $\mathrm{Rf}=0.76$ (hexane/ethyl acetate 10:1 v/v); IR (ATR) $\bar{v}_{\max } / \mathrm{cm}^{-1} 3286,3025,2964,2938,2116,1436,1351$, 1173, 1012, 974, 938, 832, 656, 528; ' $\mathrm{H}$ NMR (300 MHz, $\left.\mathrm{CDCl}_{3}\right) \delta 1.93$ (quint, 2H, J6.1 Hz), 1.99 (t, 1H, J 2.7 Hz), $2.33\left(\mathrm{dt}, 2 \mathrm{H}, J_{1} 6.1 \mathrm{~Hz}, J_{2} 2.7 \mathrm{~Hz}\right), 3.00(\mathrm{~s}, 3 \mathrm{H}), 4.32$ (t, $2 \mathrm{H}, J 6.1 \mathrm{~Hz}) ;{ }^{13} \mathrm{C} \mathrm{NMR}\left(75 \mathrm{MHz}, \mathrm{CDCl}_{3}\right) \delta 14.6,27.7$, 37.1, 68.2, 69.7, 82.0; MS-EI, $m / z(\%): 162(0) \mathrm{C}_{6} \mathrm{H}_{10} \mathrm{O}_{3} \mathrm{~S}$ $\left[\mathrm{M}^{+\bullet}\right], 122$ (3), 109 (5), 97 (4), 83 (29), 79 (51), 66 (100), 55 (19), 40 (22).

Synthesis of (Z)-3,7-dimethyl-1-(pent-4-yn-1-yloxy)octa2,6-diene (8)

A $125 \mathrm{~mL}$ round-bottom flask was charged with sodium hydride $(480 \mathrm{mg}, 20.0 \mathrm{mmol})$ and $10 \mathrm{~mL}$ of anhydrous THF. The mixture was warmed to $40{ }^{\circ} \mathrm{C}$ followed by slow addition of (2Z)-3,7-dimethylocta-2,6-dien-1-ol (nerol, compound 6 in Scheme 1) (1.54 g, $10.0 \mathrm{mmol}$ ) dissolved in anhydrous THF $(2 \mathrm{~mL})$. The system was maintained at $40{ }^{\circ} \mathrm{C}$ and magnetically stirred for $15 \mathrm{~h}$. After that, a solution of compound 5 ( $2.43 \mathrm{~g}, 15.0 \mathrm{mmol})$ in THF $(2 \mathrm{~mL})$ was added slowly. The resulting reaction mixture was maintained at $40{ }^{\circ} \mathrm{C}$ and magnetically stirred for additional $8 \mathrm{~h}$. Upon completion of the reaction as revealed by TLC analysis, $20 \mathrm{~mL}$ of $2 \mathrm{~mol} \mathrm{~L}^{-1} \mathrm{HCl}$ aqueous solution were added. The aqueous layer was extracted with ethyl acetate $(3 \times 20 \mathrm{~mL})$. The organic extracts were combined and the resulting organic phase was washed with water $(20 \mathrm{~mL})$, brine $(20 \mathrm{~mL})$, dried over $\mathrm{MgSO}_{4}$, filtered and concentrated under reduced pressure. The crude product was purified by silica gel column chromatography eluted with hexane/ethyl acetate $(10: 1 \mathrm{v} / \mathrm{v})$. Compound $\mathbf{8}$ was obtained as brown oil in $72 \%$ yield $(1.60 \mathrm{~g}, 7.20 \mathrm{mmol})$. TLC: $\mathrm{Rf}=0.55$ (hexane/ ethyl acetate/dichloromethane 10:1:3 v/v); IR (ATR) $\bar{v}_{\text {max }} / \mathrm{cm}^{-1} 3305,2963,2924,2856,2117,1668,1445$, 1376, 1104, 1072, 828, 631; ${ }^{1} \mathrm{H}$ NMR (300 MHz, $\left.\mathrm{CDCl}_{3}\right)$ $\delta 1.60(\mathrm{~s}, 3 \mathrm{H}), 1.67$ (s, 3H), $1.74(\mathrm{~s}, 3 \mathrm{H}), 1.78$ (quint, $2 \mathrm{H}$, $J 6.6 \mathrm{~Hz}), 1.92(\mathrm{t}, 1 \mathrm{H}, J 2.6 \mathrm{~Hz}), 2.07(\mathrm{~s}, 4 \mathrm{H}), 2.28(\mathrm{dt}, 2 \mathrm{H}$, $\left.J_{1} 6.6 \mathrm{~Hz}, J_{2} 2.6 \mathrm{~Hz}\right), 3.49(\mathrm{t}, 2 \mathrm{H}, J 6.6 \mathrm{~Hz}), 3.94(\mathrm{~d}, 2 \mathrm{H}$, $J 6.6 \mathrm{~Hz}), 5.09$ (brs, 1H), 5.35 (t, 1H, J 6.6 Hz); ${ }^{13} \mathrm{C}$ NMR $\left(75 \mathrm{MHz}, \mathrm{CDCl}_{3}\right) \delta 15.3,17.6,23.4,25.7,26.7,28.7,32.2$, 67.1, 68.3, 68.4, 121.9, 123.8, 131.9, 140.3; MS-EI, $\mathrm{m} / \mathrm{z}$ (\%): 220 (1) $\mathrm{C}_{15} \mathrm{H}_{24} \mathrm{O}\left[\mathrm{M}^{+\bullet}\right], 205$ (4), 177 (3), 163 (8), 149 (17), 135 (19), 121 (60), 107 (21), 93 (100), 80 (51), 69 (90), 41 (99).

Synthesis of (Z)-1-benzyl-4-(((3,7-dimethylocta-2,6-dien1-yl)oxy)methyl)-1H-1,2,3-triazole (9a)

Benzyl azide (3a, $100 \mathrm{mg}, 0.750 \mathrm{mmol}$ ) was added to a $10 \mathrm{~mL}$ round-bottom flask containing $1.50 \mathrm{~mL}$ of dichloromethane, $1.50 \mathrm{~mL}$ of water, $60.0 \mathrm{mg}(0.300 \mathrm{mmol})$ of sodium ascorbate and $144 \mathrm{mg}(0.750 \mathrm{mmol})$ of compound 7. Then, it was added $38.0 \mathrm{mg}(0.150 \mathrm{mmol})$ of $\mathrm{CuSO}_{4} \cdot 5 \mathrm{H}_{2} \mathrm{O}$. The reaction mixture was vigorously stirred at room temperature for $24 \mathrm{~h}$. After this time, saturated aqueous solution of $\mathrm{Na}_{2} \mathrm{CO}_{3}$ was added and the resulting aqueous layer was extracted with ethyl acetate $(3 \times 5 \mathrm{~mL})$. The organic extracts were combined and the resulting organic layer was dried over anhydrous $\mathrm{MgSO}_{4}$, filtered and concentrated under reduced pressured. The crude product was purified by silica gel column chromatography eluted with hexane/ethyl acetate/dichloromethane (3:1:3 v/v). Compound 9a was obtained as yellow oil in 53\% yield (106 mg, $0.330 \mathrm{mmol}$ ) along with $25 \mathrm{mg}$ of unreacted compound 7. TLC: $\mathrm{Rf}=0.48$ (hexane/ethyl acetate/ dichloromethane 3:1:3 v/v); IR (ATR) $\bar{v}_{\max } / \mathrm{cm}^{-1} 3135$, 3030, 2963, 2924, 2856, 1737, 1667, 1555, 1454, 1376, 1330, 1047, 720, 582, 460; ${ }^{1} \mathrm{H}$ NMR (300 MHz, $\mathrm{CDCl}_{3}$ ) $\delta 1.57(\mathrm{~s}, 3 \mathrm{H}), 1.66(\mathrm{~s}, 3 \mathrm{H}), 1.73(\mathrm{~s}, 3 \mathrm{H}), 2.04(\mathrm{~s}, 4 \mathrm{H})$, 4.02 (d, 2H, J 6.9 Hz), 4.58 (s, 2H), 5.06 (brs, 1H), 5.34 (t, $1 \mathrm{H}, J 6.8 \mathrm{~Hz}), 5.49$ (s, 2H), 7.24-7.27 (m, 2H), 7.33-7.36 $(\mathrm{m}, 3 \mathrm{H}), 7.44(\mathrm{~s}, 1 \mathrm{H}) ;{ }^{13} \mathrm{C} \mathrm{NMR}\left(75 \mathrm{MHz}, \mathrm{CDCl}_{3}\right) \delta 17.6$, 23.4, 25.6, 26.6, 32.2, 54.1, 63.5, 66.7, 121.3, 122.2, 123.7, $128.1,128.7,129.0,131.9,134.5,141.0,145.9$; MS-EI, $m / z(\%): 325(0.3) \mathrm{C}_{20} \mathrm{H}_{27} \mathrm{~N}_{3} \mathrm{O}\left[\mathrm{M}^{+\bullet}\right], 296$ (1), 256 (32), 190 (21), 173 (34), 144 (25), 117 (5), 91 (100), 69 (25), 43 (8).

Compounds $9 \mathbf{b}-\mathbf{9 k}$ and $\mathbf{1 0 a}-\mathbf{1 0 k}$ were synthesized using similar methodology to that described for compound $\mathbf{9 a}$. The identities of these compounds are in agreement with the following data.

(Z)-4-(((3,7-Dimethylocta-2,6-dien-1-yl)oxy)methyl)1-(4-fluorobenzyl)-1H-1,2,3-triazole (9b)

Compound 9b was obtained as a yellow oil in 54\% yield (140.0 mg, $0.40 \mathrm{mmol}$ ) from 4-fluorobenzylazide (3b) (113.2 mg, $0.750 \mathrm{mmol})$ and compound 7 (144.0 mg, $0.750 \mathrm{mmol}$ ). TLC: $\mathrm{Rf}=0.40$ (hexane/ethyl acetate/ dichloromethane 3:1:3 v/v); IR (ATR) $\overline{\mathrm{v}}_{\max } / \mathrm{cm}^{-1} 3133$, 2963, 2924, 2856, 1667, 1606, 1511, 1453, 1376, 1224, 1159, 1095, 1048, 1015, 824, 788, 534, 492; ${ }^{1} \mathrm{H}$ NMR $\left(300 \mathrm{MHz}, \mathrm{CDCl}_{3}\right) \delta 1.56(\mathrm{~s}, 3 \mathrm{H}), 1.64(\mathrm{~s}, 3 \mathrm{H}), 1.71(\mathrm{~s}$, $3 \mathrm{H}), 2.03$ (s, 4H), 4.01 (d, 2H, J 6.9 Hz), 4.57 (s, 2H), 5.04 (brs, 1H), $5.33(\mathrm{t}, 1 \mathrm{H}, J 6.9 \mathrm{~Hz}), 5.46(\mathrm{~s}, 2 \mathrm{H}), 7.00-7.05(\mathrm{~m}$, $2 \mathrm{H}), 7.22-7.26(\mathrm{~m}, 2 \mathrm{H}), 7.43(\mathrm{~s}, 1 \mathrm{H}) ;{ }^{13} \mathrm{C} \mathrm{NMR}(75 \mathrm{MHz}$, $\left.\mathrm{CDCl}_{3}\right) \delta 17.6,23.5,25.7,26.6,32.2,53.3,63.5,66.8$, $116.0\left(\mathrm{~d}, J_{\mathrm{C}-\mathrm{F}} 21.6 \mathrm{~Hz}\right), 121.3,122.1,123.7,129.9\left(\mathrm{~d}, J_{\mathrm{C}-\mathrm{F}}\right.$ $8.3 \mathrm{~Hz}), 130.4$ (d, $\left.J_{\text {C-F }} 3.2 \mathrm{~Hz}\right), 132.0,141.1,146.0,162.8$ (d, $J_{\text {C-F }} 246.5 \mathrm{~Hz}$ ); MS-EI, $m / z(\%): 343(0.3) \mathrm{C}_{20} \mathrm{H}_{26} \mathrm{FN}_{3} \mathrm{O}$ [M+•], 314 (1), 274 (16), 261 (3), 208 (16), 191 (34), 162 (20), 121 (10), 109 (100), 93 (29), 83 (19), 69 (50), 53 (16), 41 (79). 
(Z)-1-(4-Chlorobenzyl)-4-(((3,7-dimethylocta-2,6-dien-1-yl) oxy)methyl)-1H-1,2,3-triazole (9c)

Compound 9c was obtained as a yellow oil in $68 \%$ yield $(152 \mathrm{mg}, 0.400 \mathrm{mmol})$ from 4-chlorobenzylazide (3c) $(125.2 \mathrm{mg}, 0.750 \mathrm{mmol})$ and compound $7(144 \mathrm{mg}$, $0.750 \mathrm{mmol}) .25 \mathrm{mg}$ of unreacted compound 7 was also recovered. TLC: $\mathrm{Rf}=0.50$ (hexane/ethyl acetate/ dichloromethane $3: 1: 3 \mathrm{v} / \mathrm{v}$ ); IR (ATR) $\bar{v}_{\max } / \mathrm{cm}^{-1} 3134$, 3027, 2963, 2923, 2855, 1737, 1666, 1597, 1492, 1456, $1376,1220,1090,1050,786,502 ;{ }^{1} \mathrm{H}$ NMR $(300 \mathrm{MHz}$ $\left.\mathrm{CDCl}_{3}\right) \delta 1.57(\mathrm{~s}, 3 \mathrm{H}), 1.65(\mathrm{~s}, 3 \mathrm{H}), 1.73(\mathrm{~s}, 3 \mathrm{H}), 2.04(\mathrm{~s}$ $4 \mathrm{H}), 4.02(\mathrm{~d}, 2 \mathrm{H}, J 6.9 \mathrm{~Hz}), 4.58$ (s, 2H), 5.06 (brs, 1H), $5.34(\mathrm{t}, 1 \mathrm{H}, J 6.9 \mathrm{~Hz}), 5.46(\mathrm{~s}, 2 \mathrm{H}), 7.19(\mathrm{~d}, 2 \mathrm{H}, J 8.4 \mathrm{~Hz})$, $7.33(\mathrm{~d}, 2 \mathrm{H}, J 8.4 \mathrm{~Hz}), 7.44(\mathrm{~s}, 1 \mathrm{H}) ;{ }^{13} \mathrm{C}$ NMR $(75 \mathrm{MHz}$, $\left.\mathrm{CDCl}_{3}\right) \delta 17.6,23.4,25.6,26.6,32.2,53.3,63.5,66.8$, 121.2, 122.1, 123.7, 129.3, 129.4, 131.9, 133.1, 134.7, 141.1, 146.1; MS-EI, $m / z(\%): 359(0.1) \mathrm{C}_{20} \mathrm{H}_{26} \mathrm{ClN}_{3} \mathrm{O}\left[\mathrm{M}^{+*}\right]$, 330 (0.4), 290 (4), 224 (9), 207 (14), 178 (5), 125 (100), 93 (15), 69 (24), 53 (9), 41 (76).

(Z)-1-(4-Bromobenzyl)-4-(((3,7-dimethylocta-2,6-dien-1-yl) oxy)methyl)-1 $H-1,2,3$-triazole (9d)

Compound 9d was obtained as a yellow oil in 67\% yield (168 mg, $0.400 \mathrm{mmol})$ from 4-bromobenzylazide (3d) $(159 \mathrm{mg}, 0.750 \mathrm{mmol})$ and compound $7(144 \mathrm{mg}$, $0.750 \mathrm{mmol}) .25 \mathrm{mg}$ of unreacted compound 7 were also recovered. TLC: $\mathrm{Rf}=0.36$ (hexane/ethyl acetate/ dichloromethane $3: 1: 3 \mathrm{v} / \mathrm{v}$ ); IR (ATR) $\overline{\mathrm{v}}_{\max } / \mathrm{cm}^{-1} 3135,3026$, 2963, 2922, 2855, 1737, 1667, 1593, 1556, 1489, 1446, 1376, 1220, 1070, 1012, 784, 490; ${ }^{1} \mathrm{H}$ NMR (300 MHz, $\mathrm{CDCl}_{3}$ ) $\delta 1.57(\mathrm{~s}, 3 \mathrm{H}), 1.65(\mathrm{~s}, 3 \mathrm{H}), 1.73(\mathrm{~s}, 3 \mathrm{H}), 2.04(\mathrm{~s}, 4 \mathrm{H}), 4.02$ (d, $2 \mathrm{H}, J 6.9 \mathrm{~Hz}), 4.58$ (s, 2H), 5.06 (brs, 1H), 5.34 (t, 1H, $J 6.9 \mathrm{~Hz}), 5.45$ (s, 2H), $7.13(\mathrm{~d}, 2 \mathrm{H}, J 8.4 \mathrm{~Hz}), 7.44(\mathrm{~s}, 1 \mathrm{H})$, 7.47 (d, $2 \mathrm{H}, J 8.4 \mathrm{~Hz}$ ); ${ }^{13} \mathrm{C}$ NMR $\left(75 \mathrm{MHz}, \mathrm{CDCl}_{3}\right) \delta 17.6$, 23.4, 25.6, 26.6, 32.2, 53.4, 63.5, 66.8, 121.2, 122.2, 122.8, 123.7, 129.6, 131.9, 132.2, 133.6, 141.1, 146.1; MS-EI, $\mathrm{m} / \mathrm{z}$ (\%): $404(0.04) \mathrm{C}_{20} \mathrm{H}_{26} \mathrm{BrN}_{3} \mathrm{O}\left[\mathrm{M}^{+*}\right], 336(4), 270$ (3), 251 (8), 169 (36), 121 (7), 93 (14), 90 (16), 69 (32), 53 (12), 41 (100).

(Z)-4-(((3,7-Dimethylocta-2,6-dien-1-yl)oxy)methyl)1-(4-iodobenzyl)-1H-1,2,3-triazole (9e)

Compound 9e was obtained as a yellow oil in 52\% yield (150 mg, $0.330 \mathrm{mmol}$ ) from 4-iodobenzylazide (3e) $(194.2 \mathrm{mg}, 0.750 \mathrm{mmol})$ and compound $7(144.0 \mathrm{mg}$, $0.750 \mathrm{mmol}$ ). TLC: $\mathrm{Rf}=0.55$ (hexane/ethyl acetate/ dichloromethane $3: 1: 3 \mathrm{v} / \mathrm{v}$ ); IR (ATR) $\bar{v}_{\max } / \mathrm{cm}^{-1} 3135$, 3022, 2963, 2923, 2855, 1704, 1690, 1667, 1556, 1485, 1446, 1375, 1220, 1048, 1008, 783, 474; ${ }^{1} \mathrm{H}$ NMR $\left(300 \mathrm{MHz}, \mathrm{CDCl}_{3}\right) \delta 1.56(\mathrm{~s}, 3 \mathrm{H}), 1.65(\mathrm{~s}, 3 \mathrm{H}), 1.72(\mathrm{~s}$, $3 \mathrm{H}), 2.04(\mathrm{~s}, 4 \mathrm{H}), 4.02(\mathrm{~d}, 2 \mathrm{H}, J 6.9 \mathrm{~Hz}), 4.57(\mathrm{~s}, 2 \mathrm{H}), 5.05$ (brs, 1H), 5.33 (t, 1H, J 6.9 Hz), 5.42 (s, 2H), 6.98 (d, 2H,
$J 8.3 \mathrm{~Hz}), 7.44$ (s, 1H), 7.66 (d, 2H, J 8.3 Hz); ${ }^{13} \mathrm{C}$ NMR $\left(75 \mathrm{MHz}, \mathrm{CDCl}_{3}\right) \delta 17.6,23.4,25.6,26.6,32.1,53.4,63.4$, 66.7, 94.4, 121.2, 122.2, 123.7, 129.8, 131.9, 134.2, 138.1, 141.0, 146.1; MS-EI, $m / z(\%): 451(0.09) \mathrm{C}_{20} \mathrm{H}_{26} \mathrm{IN}_{3} \mathrm{O}\left[\mathrm{M}^{+*}\right]$, 382 (10), 299 (7), 217 (56), 143 (13), 90 (22), 69 (42), 53 (14), 41 (100).

(Z)-4-(((3,7-Dimethylocta-2,6-dien-1-yl)oxy)methyl)1-(4-(trifluoromethoxy)benzyl)-1H-1,2,3-triazole (9f)

Compound 9 f was obtained as a yellow oil in $41 \%$ yield (127 mg, $0.310 \mathrm{mmol}$ ) from 4-trifluoromethoxybenzylazide (3f) $(163 \mathrm{mg}, 0.750 \mathrm{mmol})$ and compound $7(144.0 \mathrm{mg}$, $0.750 \mathrm{mmol}$ ). TLC: $\mathrm{Rf}=0.28$ (hexane/ethyl acetate/ dichloromethane $3: 1: 3 \mathrm{v} / \mathrm{v}$ ); IR (ATR) $\bar{v}_{\max } / \mathrm{cm}^{-1} 3133$, 2965, 2925, 2858, 1668, 1597, 1510, 1453, 1377, 1260, 1221, 1166, 1048, 1019, 922, 664, 628; ' $\mathrm{H}$ NMR $(300 \mathrm{MHz}$, $\left.\mathrm{CDCl}_{3}\right) \delta 1.57$ (s, 3H), 1.65 (s, 3H), 1.72 (s, 3H), 2.04 (s, $4 \mathrm{H}), 4.03(\mathrm{~d}, 2 \mathrm{H}, J 6.9 \mathrm{~Hz}), 4.58(\mathrm{~s}, 2 \mathrm{H}), 5.05$ (brs, 1H), $5.34(\mathrm{t}, 1 \mathrm{H}, J 6.9 \mathrm{~Hz}), 5.50(\mathrm{~s}, 2 \mathrm{H}), 7.19(\mathrm{~d}, 2 \mathrm{H}, J 8.4 \mathrm{~Hz})$, $7.28(\mathrm{~d}, 2 \mathrm{H}, J 8.4 \mathrm{~Hz}), 7.47(\mathrm{~s}, 1 \mathrm{H}) ;{ }^{13} \mathrm{C}$ NMR $(75 \mathrm{MHz}$, $\left.\mathrm{CDCl}_{3}\right) \delta 17.6,23.4,25.6,26.6,32.2,53.2,63.5,66.8$, $120.3\left(\mathrm{q}, J_{\mathrm{C}-\mathrm{F}} 256.0 \mathrm{~Hz}\right), 121.2,121.5,122.2,123.6,129.5$, 132.0, 133.3, 141.2, 146.2, 149.4; MS-EI, $m / z(\%): 409$ (1) $\mathrm{C}_{21} \mathrm{H}_{26} \mathrm{~F}_{3} \mathrm{~N}_{3} \mathrm{O}_{2}\left[\mathrm{M}^{+*}\right], 340$ (9), 327 (7), 274 (27), 257 (52), 228 (32), 188 (10), 175 (100), 136 (9), 121 (22), 109 (29), 93 (57), 80 (35), 69 (69), 53 (18), 41 (74).

(Z)-4-(((3,7-Dimethylocta-2,6-dien-1-yl)oxy)methyl)1-(4-(trifluoromethyl)benzyl)-1 H-1,2,3-triazole (9g)

Compound $9 \mathrm{~g}$ was obtained as a yellow oil in $48 \%$ yield (142 mg, $0.360 \mathrm{mmol}$ ) from 4-trifluoromethylbenzylazide $(3 \mathrm{~g})(151 \mathrm{mg}, 0.750 \mathrm{mmol})$ and compound $7(144 \mathrm{mg}$, $0.750 \mathrm{mmol}$ ). TLC: $\mathrm{Rf}=0.29$ (hexane/ethyl acetate/ dichloromethane $3: 1: 3 \mathrm{v} / \mathrm{v}$ ); IR (ATR) $\bar{v}_{\max } / \mathrm{cm}^{-1} 3135,2965$, 2926, 2857, 1703, 1668, 1621, 1448, 1325, 1251, 1166, 1127, 1067, 1018, 824, 790, 622; ${ }^{1} \mathrm{H}$ NMR (300 MHz, $\mathrm{CDCl}_{3}$ ) $\delta 1.56(\mathrm{~s}, 3 \mathrm{H}), 1.64(\mathrm{~s}, 3 \mathrm{H}), 1.72(\mathrm{~s}, 3 \mathrm{H}), 2.03(\mathrm{~s}, 4 \mathrm{H}), 4.03$ (d, $2 \mathrm{H}, J 6.9 \mathrm{~Hz}), 4.59$ (s, 2H), 5.04 (brs, 1H), $5.33(\mathrm{t}, 1 \mathrm{H}$, $J 6.9 \mathrm{~Hz}), 5.56(\mathrm{~s}, 2 \mathrm{H}), 7.35(\mathrm{~d}, 2 \mathrm{H}, J 8.4 \mathrm{~Hz}), 7.48(\mathrm{~s}, 1 \mathrm{H})$, $7.60(\mathrm{~d}, 2 \mathrm{H}, J 8.4 \mathrm{~Hz}) ;{ }^{13} \mathrm{C}$ NMR $\left(75 \mathrm{MHz}, \mathrm{CDCl}_{3}\right) \delta 17.6$, 23.5, 25.7, 26.6, 32.2, 53.4, 63.5, 66.8, 121.2, 122.4, 123.7, $124.6\left(\mathrm{q}, J_{\mathrm{C}-\mathrm{F}} 270.0 \mathrm{~Hz}\right), 126.0\left(\mathrm{q}, J_{\mathrm{C}-\mathrm{F}} 3.7 \mathrm{~Hz}\right), 128.2,130.9$ (q, $\left.\mathrm{J}_{\mathrm{C}-\mathrm{F}} 32.4 \mathrm{~Hz}\right), 132.0,138.6,141.2,146.3$; MS-EI, $\mathrm{m} / \mathrm{z}(\%)$ : $393(0.9) \mathrm{C}_{21} \mathrm{H}_{26} \mathrm{~F}_{3} \mathrm{~N}_{3} \mathrm{O}\left[\mathrm{M}^{+*}\right], 364$ (2), 324 (34), 311 (5), 258 (33), 241 (14), 212 (45), 172 (24), 159 (100), 121 (23), 109 (41), 93 (60), 80 (33), 69 (78), 53 (24), 41 (99).

(Z)-4-(((3,7-Dimethylocta-2,6-dien-1-yl)oxy)methyl)1-(4-methoxybenzyl)-1H-1,2,3-triazole (9h)

Compound $9 \mathrm{~h}$ was obtained as a yellow oil in $65 \%$ yield (140 mg, $0.390 \mathrm{mmol}$ ) from 4-methoxybenzylazide 
(3h) $(122 \mathrm{mg}, 0.750 \mathrm{mmol})$ and compound 7 (144 mg, $0.750 \mathrm{mmol}) .28 \mathrm{mg}$ of unreacted compound 7 was also recovered. TLC: $\mathrm{Rf}=0.49$ (hexane/ethyl acetate/ dichloromethane $3: 1: 3 \mathrm{v} / \mathrm{v}$ ); IR (ATR) $\bar{v}_{\max } / \mathrm{cm}^{-1} 3134$, 3067, 2962, 2928, 2856, 1667, 1612, 1585, 1514, 1453, $1375,1249,1177,1047,814,786,554,516 ;{ }^{1} \mathrm{H}$ NMR $\left(300 \mathrm{MHz}, \mathrm{CDCl}_{3}\right) \delta 1.56(\mathrm{~s}, 3 \mathrm{H}), 1.65(\mathrm{~s}, 3 \mathrm{H}), 1.72(\mathrm{~s}$, $3 \mathrm{H}), 2.03(\mathrm{~s}, 4 \mathrm{H}), 3.78(\mathrm{~s}, 3 \mathrm{H}), 4.01(\mathrm{~d}, 2 \mathrm{H}, J 6.6 \mathrm{~Hz}), 4.56$ (s, 2H), 5.05 (brs, 1H), 5.33 (t, 1H, J 6.6 Hz), $5.42(\mathrm{~s}, 2 \mathrm{H})$, $6.87(\mathrm{~d}, 2 \mathrm{H}, J 8.7 \mathrm{~Hz}), 7.21(\mathrm{~d}, 2 \mathrm{H}, J 8.7 \mathrm{~Hz}), 7.40(\mathrm{~s}, 1 \mathrm{H})$; ${ }^{13} \mathrm{C} \mathrm{NMR}\left(75 \mathrm{MHz}, \mathrm{CDCl}_{3}\right) \delta 17.6,23.5,25.7,26.6,32.2$, 53.6, 55.3, 63.5, 66.7, 114.4, 121.3, 122.0, 123.7, 126.5, 129.7, 131.9, 141.0, 145.8, 159.9; MS-EI, $\mathrm{m} / \mathrm{z}(\%): 355$ (0.18) $\mathrm{C}_{21} \mathrm{H}_{29} \mathrm{~N}_{3} \mathrm{O}_{2}\left[\mathrm{M}^{+*}\right], 326(0.3), 286$ (6), 273 (1), 220 (3), 203 (4), 134 (3), 121 (100), 107 (3), 91 (12), 77 (17), 69 (27), 53 (10), 41 (60).

(Z)-4-(((3,7-Dimethylocta-2,6-dien-1-yl)oxy)methyl)1-(4-nitrobenzyl)-1H-1,2,3-triazole (9i)

Compound $9 \mathbf{i}$ was obtained as a yellow oil in $40 \%$ yield $(96.0 \mathrm{mg}, 0.260 \mathrm{mmol})$ from 4-nitrobenzylazide (3i) $(134 \mathrm{mg}, 0.750 \mathrm{mmol})$ and compound $7(144 \mathrm{mg}$, $0.750 \mathrm{mmol}$ ). An amount of $40 \mathrm{mg}$ of unreacted compound 7 was also recovered. TLC: $\mathrm{Rf}=0.34$ (hexane/ethyl acetate/ dichloromethane $3: 1: 3 \mathrm{v} / \mathrm{v}$ ); IR (ATR) $\bar{v}_{\max } / \mathrm{cm}^{-1} 3137$, 3079, 2964, 2925, 2856, 1667, 1523, 1494, 1346, 1222, 1047, 804, 726; ' $\mathrm{H}$ NMR (300 MHz, $\left.\mathrm{CDCl}_{3}\right) \delta 1.57$ (s, $3 \mathrm{H}), 1.65(\mathrm{~s}, 3 \mathrm{H}), 1.73(\mathrm{~s}, 3 \mathrm{H}), 2.05(\mathrm{~s}, 4 \mathrm{H}), 4.04(\mathrm{~d}, 2 \mathrm{H}$, $J 6.9 \mathrm{~Hz}), 4.61$ (s, 2H), 5.05 (brs, 1H), 5.34 (t, $1 \mathrm{H}, J 6.9 \mathrm{~Hz})$, $5.62(\mathrm{~s}, 2 \mathrm{H}), 7.39$ (d, 2H, J 8.4 Hz), $7.53(\mathrm{~s}, 1 \mathrm{H}), 8.20$ (d, $2 \mathrm{H}, J 8.4 \mathrm{~Hz}) ;{ }^{13} \mathrm{C}$ NMR $\left(75 \mathrm{MHz}, \mathrm{CDCl}_{3}\right) \delta 17.6,23.5$, 25.7, 26.7, 32.2, 53.1, 63.5, 66.9, 121.1, 122.5, 123.7, 124.3, 126.6, 132.0, 141.3, 141.6, 146.6, 147.9; MS-EI, $m / z(\%): 370(0.12) \mathrm{C}_{20} \mathrm{H}_{26} \mathrm{~N}_{4} \mathrm{O}_{3}\left[\mathrm{M}^{+*}\right], 301$ (5), 235 (4), 218 (14), 189 (3), 136 (12), 121 (6), 106 (20), 93 (17), 78 (28), 69 (41), 53 (16), 41 (100).

(Z)-1-(3,4-Difluorobenzyl)-4-(((3,7-dimethylocta-2,6-dien1-yl)oxy)methyl)-1 $H$-1,2,3-triazole (9j)

Compound $9 \mathbf{j}$ was obtained as a yellow oil in $45 \%$ yield ( $123 \mathrm{mg}, 0.340 \mathrm{mmol}$ ) from 3,4-difluorobenzylazide (3j) $(127 \mathrm{mg}, 0.750 \mathrm{mmol})$ and compound $7(144 \mathrm{mg}$, $0.750 \mathrm{mmol}$ ). TLC: $\mathrm{Rf}=0.36$ (hexane/ethyl acetate/ dichloromethane $3: 1: 3 \mathrm{v} / \mathrm{v}$ ); IR (ATR) $\bar{v}_{\max } / \mathrm{cm}^{-1} 3135$, 2964, 2925, 2857, 1715, 1669, 1611, 1521, 1439, 1376, 1286, 1214, 1116, 1048, 781, 576, 526, 452; ${ }^{1} \mathrm{H}$ NMR $\left(300 \mathrm{MHz}, \mathrm{CDCl}_{3}\right) \delta 1.56(\mathrm{~s}, 3 \mathrm{H}), 1.64(\mathrm{~s}, 3 \mathrm{H}), 1.71(\mathrm{~s}, 3 \mathrm{H})$, 2.03 (s, 4H), 4.02 (d, 2H, J $6.9 \mathrm{~Hz}), 4.58$ (s, 2H), 5.04 (brs, $1 \mathrm{H}), 5.33(\mathrm{t}, 1 \mathrm{H}, J 6.9 \mathrm{~Hz}), 5.44(\mathrm{~s}, 2 \mathrm{H}), 6.98-7.18(\mathrm{~m}, 3 \mathrm{H})$, $7.48(\mathrm{~s}, 1 \mathrm{H}) ;{ }^{13} \mathrm{C} \mathrm{NMR}\left(75 \mathrm{MHz}, \mathrm{CDCl}_{3}\right) \delta 17.6,23.5,25.7$, 26.6, 32.2, 52.9, 63.5, 66.8, 117.1-117.3 (m), 117.8-118.1 (m), 121.2, 122.2, 123.7, 124.1-124.3(m), 131.5-131.6(m), 132.0, 146.3, 148.7-148.9 (m), 152.0-152.2 (m); MS-EI, $m / z(\%): 361(0.17) \mathrm{C}_{20} \mathrm{H}_{25} \mathrm{~F}_{2} \mathrm{~N}_{3} \mathrm{O}\left[\mathrm{M}^{+*}\right], 344$ (0.07), 292 (9), 226 (9), 209 (20), 180 (15), 140 (7), 127 (100), 107 (8), 93 (24), 80 (11), 69 (49), 53 (17), 41 (98).

(Z)-1-(2,5-Dichlorobenzyl)-4-(((3,7-dimethylocta-2,6-dien1-yl)oxy)methyl)-1 $H$-1,2,3-triazole (9k)

Compound 9k was obtained as a yellow oil in $76 \%$ yield (118 mg, $0.300 \mathrm{mmol}$ ) from 2,5-dichlorobenzylazide (3k) $(101 \mathrm{mg}, 0.500 \mathrm{mmol})$ and compound $7(96.0 \mathrm{mg}$, $0.500 \mathrm{mmol}$ ). $25.0 \mathrm{mg}$ of unreacted compound 7 was also recovered. TLC: $\mathrm{Rf}=0.66$ (hexane/ethyl acetate/ dichloromethane $3: 1: 3 \mathrm{v} / \mathrm{v}$ ); IR (ATR) $\bar{v}_{\max } / \mathrm{cm}^{-1} 3138$, 3088, 2964, 2925, 2857, 1720, 1668, 1466, 1376, 1223, 1099, 1048, 816, 562; ${ }^{1} \mathrm{H}$ NMR (300 MHz, $\left.\mathrm{CDCl}_{3}\right) \delta 1.57$ (s, 3H), 1.65 (s, 3H), 1.73 (s, 3H), 2.05 (s, 4H), 4.04 (d, $2 \mathrm{H}, J 6.9 \mathrm{~Hz}), 4.61(\mathrm{~s}, 2 \mathrm{H}), 5.06$ (brs, $1 \mathrm{H}), 5.35(\mathrm{t}, 1 \mathrm{H}$, $J 6.9 \mathrm{~Hz}), 5.60(\mathrm{~s}, 2 \mathrm{H}), 7.15(\mathrm{~d}, 1 \mathrm{H}, J 2.1 \mathrm{~Hz}), 7.27(\mathrm{dd}$, $\left.1 \mathrm{H}, J_{1} 8.5 \mathrm{~Hz}, J_{2} 2.1 \mathrm{~Hz}\right), 7.35$ (d, 1H, J 8.5 Hz), 7.57 (s, $1 \mathrm{H}) ;{ }^{13} \mathrm{C} \mathrm{NMR}\left(75 \mathrm{MHz}, \mathrm{CDCl}_{3}\right) \delta 17.6,23.5,25.7,26.6$, 32.2, 50.9, 63.5, 66.8, 121.2, 122.6, 123.7, 130.1, 131.0, 131.6, 132.0, 133.5, 134.0, 141.2, 146.1; MS-EI, $\mathrm{m} / \mathrm{z}(\%)$ : $394(0.2) \mathrm{C}_{20} \mathrm{H}_{25} \mathrm{Cl}_{2} \mathrm{~N}_{3} \mathrm{O}\left[\mathrm{M}^{+*}\right], 364$ (0.8), 324 (14), 258 (16), 241 (31), 212 (11), 177 (5), 160 (4), 121 (11), 93 (27), 53 (11), 41 (100).

(Z)-1-Benzyl-4-(3-((3,7-dimethylocta-2,6-dien-1-yl)oxy) propyl)-1H-1,2,3-triazole (10a)

Compound 10a was obtained as a yellow oil in $76 \%$ yield (203 mg, $0.570 \mathrm{mmol}$ ) from compounds $\mathbf{3 a}$ (100 mg, $0.750 \mathrm{mmol})$ and $8(165 \mathrm{mg}, 0.750 \mathrm{mmol}) .35 \mathrm{mg}$ of unreacted compound $\mathbf{8}$ was also recovered. TLC: $\mathrm{Rf}=0.47$ (hexane/ethyl acetate/dichloromethane 3:1:3 v/v); IR (ATR) $\bar{v}_{\max } / \mathrm{cm}^{-1} 3133,3064,3031,2959,2924,2855,1714$, 1667, 1552, 1454, 1376, 1217, 1102, 726, 460; ${ }^{1} \mathrm{H}$ NMR $\left(300 \mathrm{MHz}, \mathrm{CDCl}_{3}\right) \delta 1.57(\mathrm{~s}, 3 \mathrm{H}), 1.64(\mathrm{~s}, 3 \mathrm{H}), 1.71(\mathrm{~s}$, $3 \mathrm{H}$ ), 1.90 (quint, $2 \mathrm{H}, J 6.3 \mathrm{~Hz}$ ), $2.04(\mathrm{~s}, 4 \mathrm{H}), 2.75(\mathrm{t}, 2 \mathrm{H}$, $J 6.3 \mathrm{~Hz}$ ), 3.41 (t, 2H, J $6.3 \mathrm{~Hz}$ ), 3.90 (d, 2 H, J $6.9 \mathrm{~Hz}), 5.06$ (brs, 1H), $5.31(\mathrm{t}, 1 \mathrm{H}, J 6.9 \mathrm{~Hz}), 5.46(\mathrm{~s}, 2 \mathrm{H}), 7.18(\mathrm{~s}, 1 \mathrm{H})$, 7.21-7.23 (m, 2H), 7.32-7.34 (m, 3H); ${ }^{13} \mathrm{C}$ NMR $(75 \mathrm{MHz}$, $\left.\mathrm{CDCl}_{3}\right) \delta 17.5,22.3,23.4,25.6,26.6,29.3,32.1,53.9,66.9$, 69.1, 120.7, 121.8, 123.8, 127.8, 128.5, 128.9, 131.8, 134.9, 140.1, 148.1; MS-EI, $m / z(\%): 353(0.15) \mathrm{C}_{22} \mathrm{H}_{31} \mathrm{~N}_{3} \mathrm{O}\left[\mathrm{M}^{+*}\right]$, 310 (0.11), 285 (3), 218 (30), 200 (16), 187 (2), 173 (55), 121 (4), 91 (100), 69 (35), 53 (15), 41 (75).

(Z)-4-(3-((3,7-Dimethylocta-2,6-dien-1-yl)oxy)propyl)1-(4-fluorobenzyl)-1 H-1,2,3-triazole (10b)

Compound 10b was obtained as a yellow oil in $42 \%$ yield ( $96.0 \mathrm{mg}, 0.260 \mathrm{mmol}$ ) from compounds $\mathbf{3 b}$ (113 mg, 
$0.750 \mathrm{mmol})$ and $8(165 \mathrm{mg}, 0.750 \mathrm{mmol}) .50 \mathrm{mg}$ of unreacted compound $\mathbf{8}$ was also recovered. TLC: $\mathrm{Rf}=0.32$ (hexane/ethyl acetate/dichloromethane 3:1:3 v/v). IR (ATR) $\bar{v}_{\max } / \mathrm{cm}^{-1} 3133,2955,2923,2854,1715,1667,1606,1511$, 1454, 1376, 1225, 1158, 1101, 1046, 824, 784, 534, 490; ${ }^{1} \mathrm{H}$ NMR (300 MHz, CDCl $) \delta 1.57$ (s, 3H), 1.65 (s, 3H), $1.72(\mathrm{~s}, 3 \mathrm{H}), 1.91$ (quint, $2 \mathrm{H}, J 6.3 \mathrm{~Hz}), 2.04(\mathrm{~s}, 4 \mathrm{H}), 2.76(\mathrm{t}$, $2 \mathrm{H}, J 6.3 \mathrm{~Hz}), 3.42$ (t, 2H, J 6.3 Hz), 3.90 (d, 2H, J $6.9 \mathrm{~Hz})$, 5.06 (brs, 1H), $5.31(\mathrm{t}, 1 \mathrm{H}, J 6.9 \mathrm{~Hz}), 5.44(\mathrm{~s}, 2 \mathrm{H}), 7.00-7.06$ (m, 2H), 7.19-7.24 (m, 3H); ${ }^{13} \mathrm{C}$ NMR $\left(75 \mathrm{MHz}, \mathrm{CDCl}_{3}\right)$ $\delta$ 17.6, 22.4, 23.4, 25.7, 26.7, 29.4, 32.2, 53.2, 67.0, 69.1, $116.0\left(\mathrm{~d}, J_{\mathrm{C}-\mathrm{F}} 21.6 \mathrm{~Hz}\right), 120.6,121.9,123.8,129.7\left(\mathrm{~d}, J_{\mathrm{C}-\mathrm{F}}\right.$ $8.2 \mathrm{~Hz}), 130.8\left(\mathrm{~d}, J_{\mathrm{C}-\mathrm{F}} 3.2 \mathrm{~Hz}\right), 131.9,140.2,148.3,162.8$ (d, $J_{\text {C- } \mathrm{F}} 246.4 \mathrm{~Hz}$ ); MS-EI, $m / z(\%): 371(0.04) \mathrm{C}_{22} \mathrm{H}_{30} \mathrm{FN}_{3} \mathrm{O}$ $\left[\mathrm{M}^{+*}\right], 302$ (0.6), 236 (9), 218 (5), 191 (20), 162 (1), 121 (2), 109 (100), 93 (7), 83 (11), 69 (31), 53 (11), 41 (61).

(Z)-1-(4-Chlorobenzyl)-4-(3-((3,7-dimethylocta-2,6-dien1-yl)oxy)propyl)-1H-1,2,3-triazole (10c)

Compound 10c was obtained as a yellow oil in $81 \%$ yield (195 mg, $0.500 \mathrm{mmol}$ ) from compounds $3 c$ (125 mg, $0.750 \mathrm{mmol})$ and 8 (165 $\mathrm{mg}, 0.750 \mathrm{mmol}) .30 \mathrm{mg}$ of unreacted compound $\mathbf{8}$ was also recovered. TLC: $\mathrm{Rf}=0.33$ (hexane/ethyl acetate/dichloromethane 3:1:3 v/v); IR (ATR) $\bar{v}_{\max } / \mathrm{cm}^{-1} 3135,2956,2924,2854,1720,1667,1597,1552$, 1492, 1453, 1376, 1217, 1099, 1047, 807, 501; ${ }^{1} \mathrm{H}$ NMR $\left(300 \mathrm{MHz}, \mathrm{CDCl}_{3}\right) \delta 1.59(\mathrm{~s}, 3 \mathrm{H}), 1.66(\mathrm{~s}, 3 \mathrm{H}), 1.73(\mathrm{~s}$, $3 \mathrm{H}$ ), 1.92 (quint, 2H, J $6.3 \mathrm{~Hz}$ ), 2.05 (s, 4H), 2.77 (t, 2H, $J 6.3 \mathrm{~Hz}), 3.43$ (t, 2H, J $6.3 \mathrm{~Hz}), 3.92$ (d, 2H, J $6.9 \mathrm{~Hz})$, 5.08 (brs, 1H), 5.32 (t, 1H, J $6.9 \mathrm{~Hz}), 5.45$ (s, 2H), 7.17 (d, 2H, J $8.4 \mathrm{~Hz}), 7.19$ (s, 1H), 7.34 (d, 2H, J $8.4 \mathrm{~Hz}$ ); ${ }^{13} \mathrm{C} \mathrm{NMR}\left(75 \mathrm{MHz}, \mathrm{CDCl}_{3}\right) \delta 17.6,22.4,23.5,25.7,26.7$, 29.4, 32.2, 53.2, 67.0, 69.1, 120.6, 121.9, 123.8, 129.2, 131.9, 133.4, 134.6, 140.2, 148.4; MS-EI, $m / z(\%): 388$ (0.2) $\mathrm{C}_{22} \mathrm{H}_{30} \mathrm{ClN}_{3} \mathrm{O}\left[\mathrm{M}^{+\bullet}\right], 318$ (0.6), 252 (12), 234 (7), 207 (22), 125 (100), 89 (10), 69 (29), 53 (11), 41 (75).

(Z)-1-(4-Bromobenzyl)-4-(3-((3,7-dimethylocta-2,6-dien1-yl)oxy)propyl)-1H-1,2,3-triazole (10d)

Compound 10d was obtained as a yellow oil in $68 \%$ yield (168 mg, $0.390 \mathrm{mmol}$ ) from compounds $3 d$ (159 mg, $0.750 \mathrm{mmol})$ and 8 (165 mg, $0.750 \mathrm{mmol}) .40 \mathrm{mg}$ of unreacted compound $\mathbf{8}$ was also recovered. TLC: $\mathrm{Rf}=0.31$ (hexane/ethyl acetate/dichloromethane 3:1:3 v/v); IR (ATR) $\overline{\mathrm{v}}_{\max } / \mathrm{cm}^{-1} 3136,2959,2926,2856,2358,1713,1667,1592$, 1552, 1489, 1445, 1376, 1218, 1070, 1012, 804, 668, 622, 478; ${ }^{1} \mathrm{H}$ NMR (300 MHz, $\left.\mathrm{CDCl}_{3}\right) \delta 1.57$ (s, 3H), 1.64 (s, 3H), 1.71 (s, 3H), 1.91 (quint, 2H, J $6.3 \mathrm{~Hz}$ ), 2.04 (s, $4 \mathrm{H}), 2.75$ (t, 2H, J 6.3 Hz), 3.41 (t, 2H, J $6.3 \mathrm{~Hz}), 3.90$ (d, $2 \mathrm{H}, J 6.9 \mathrm{~Hz}), 5.06$ (brs, 1H), 5.31 (t, 1H, J 6.9 Hz), 5.42 (s, 2H), 7.10 (d, 2H, J 8.4 Hz), 7.19 (s, 1H), 7.46 (d, 2H,
$J$ 8.4 Hz); ${ }^{13} \mathrm{C}$ NMR $\left(75 \mathrm{MHz}, \mathrm{CDCl}_{3}\right) \delta 17.6,22.4,23.5$, 25.7, 26.7, 29.4, 32.2 , 53.2, 67.0, 69.1, 120.7, 121.9, 122.7, 123.8, 129.5, 131.9, 132.2, 134.0, 140.2, 148.4; MS-EI, $m / z$ (\%): $432(0.04) \mathrm{C}_{22} \mathrm{H}_{30} \mathrm{BrN}_{3} \mathrm{O}\left[\mathrm{M}^{+\bullet}\right], 363$ (0.6), $296(9), 280$ (5), 253 (15), 169 (53), 121 (4), 90 (26), 80 (8), 69 (54), 53 (17), 41 (100).

(Z)-4-(3-((3,7-Dimethylocta-2,6-dien-1-yl)oxy)propyl)1-(4-iodobenzyl)-1H-1,2,3-triazole (10e)

Compound 10e was obtained as a yellow oil in $81 \%$ yield (230 mg, $0.480 \mathrm{mmol}$ ) from compounds $\mathbf{3 e}$ (194 mg, $0.750 \mathrm{mmol}$ ) and 8 (165 mg, $0.750 \mathrm{mmol}) .35 \mathrm{mg}$ of unreacted compound $\mathbf{8}$ was also recovered. TLC: $\mathrm{Rf}=0.30$ (hexane/ethyl acetate/dichloromethane 3:1:3 v/v); IR (ATR) $\bar{v}_{\max } / \mathrm{cm}^{-1} 3132,3075,2958,2923,2854,1715$, 1667, 1590, 1550, 1485, 1446, 1376, 1217, 1102, 1007, 812, 474; ${ }^{1} \mathrm{H}$ NMR $\left(300 \mathrm{MHz}, \mathrm{CDCl}_{3}\right) \delta 1.58(\mathrm{~s}, 3 \mathrm{H}), 1.65$ (s, 3H), 1.72 (s, 3H), 1.91 (quint, 2H, J 6.3 Hz), 2.05 (s, $4 \mathrm{H}), 2.77(\mathrm{t}, 2 \mathrm{H}, J 6.3 \mathrm{~Hz}), 3.42(\mathrm{t}, 2 \mathrm{H}, J 6.3 \mathrm{~Hz}), 3.91(\mathrm{~d}$, 2H, J $6.9 \mathrm{~Hz}), 5.07$ (brs, 1H), 5.31 (t, 1H, J 6.9 Hz), 5.41 (s, 2H), 6.96 (d, 2H, J 8.4 Hz), 7.19 (s, 1H), 7.67 (d, 2H, $J 8.4 \mathrm{~Hz}) ;{ }^{13} \mathrm{C}$ NMR $\left(75 \mathrm{MHz}, \mathrm{CDCl}_{3}\right) \delta 17.6,22.3,23.4$, 25.7, 26.7, 29.4, 32.2, 53.3, 67.0, 69.1, 94.3, 120.7, 121.9, 123.8, 129.6, 131.8, 134.6, 138.1, 140.2, 148.4; MS-EI, $m / z(\%): 479(0.3) \mathrm{C}_{22} \mathrm{H}_{30} \mathrm{IN}_{3} \mathrm{O}\left[\mathrm{M}^{+*}\right], 411$ (2), 344 (14), 326 (5), 299 (17), 217 (76), 121 (6), 90 (26), 69 (43), 53 (16), 41 (100).

(Z)-4-(3-((3,7-Dimethylocta-2,6-dien-1-yl)oxy)propyl)1-(4-(trifluoromethoxy)benzyl)-1H-1,2,3-triazole (10f)

Compound 10f was obtained as a yellow oil in $91 \%$ yield (299 mg, $0.680 \mathrm{mmol}$ ) from compounds $3 \mathbf{f}$ (163 mg, $0.750 \mathrm{mmol}$ ) and $\mathbf{8}$ (165 mg, $0.750 \mathrm{mmol})$. TLC: Rf = 0.26 (hexane/ethyl acetate/dichloromethane 3:1:3 v/v); IR (ATR) $\overline{\mathrm{v}}_{\max } / \mathrm{cm}^{-1} 3133,3074,2961,2928,2857,1713,1667,1510$, 1446, 1372, 1260, 1221, 1166, 1104, 819, 610; ${ }^{1} \mathrm{H}$ NMR $\left(300 \mathrm{MHz}, \mathrm{CDCl}_{3}\right) \delta 1.56(\mathrm{~s}, 3 \mathrm{H}), 1.64(\mathrm{~s}, 3 \mathrm{H}), 1.71(\mathrm{~s}$, $3 \mathrm{H}), 1.91$ (quint, $2 \mathrm{H}, J 6.3 \mathrm{~Hz}), 2.03(\mathrm{~s}, 4 \mathrm{H}), 2.76(\mathrm{t}, 2 \mathrm{H}$, $J 6.3 \mathrm{~Hz}), 3.42(\mathrm{t}, 2 \mathrm{H}, J 6.3 \mathrm{~Hz}), 3.90$ (d, 2H, J 6.9 Hz), 5.06 (brs, 1H), $5.31(\mathrm{t}, 1 \mathrm{H}, J 6.9 \mathrm{~Hz}), 5.47(\mathrm{~s}, 2 \mathrm{H}), 7.17-7.27(\mathrm{~m}$, $5 \mathrm{H}) ;{ }^{13} \mathrm{C} \mathrm{NMR}\left(75 \mathrm{MHz}, \mathrm{CDCl}_{3}\right) \delta 17.6,22.3,23.4,25.6$, 26.6, 29.4, 32.2, 53.0, 67.0, 69.1, 120.3 (q, J 256.0 Hz), $120.8,121.4,121.8,123.8,129.3,131.9,133.7,140.2$, 148.4, 149.3; MS-EI, $m / z(\%): 437(0.1) \mathrm{C}_{23} \mathrm{H}_{30} \mathrm{~F}_{3} \mathrm{~N}_{3} \mathrm{O}_{2}$ $\left[\mathrm{M}^{+\bullet}\right], 369$ (1), 302 (22), 284 (14), 257 (40), 175 (100), 121 (7), 109 (24), 93 (19), 80 (10), 69 (52), 53 (14), 41 (73).

(Z)-4-(3-((3,7-Dimethylocta-2,6-dien-1-yl)oxy)propyl)1-(4-(trifluoromethyl)benzyl)-1 $\mathrm{H}$-1,2,3-triazole (10g)

Compound $\mathbf{1 0 g}$ was obtained as a yellow oil in $75 \%$ yield (239 mg, $0.560 \mathrm{mmol}$ ) from compounds $3 \mathrm{~g}$ (151 mg, 
$0.750 \mathrm{mmol})$ and $\mathbf{8}(165 \mathrm{mg}, 0.75 \mathrm{mmol})$. TLC: $\mathrm{Rf}=0.31$ (hexane/ethyl acetate/dichloromethane 3:1:3 v/v); IR (ATR) $\bar{v}_{\max } / \mathrm{cm}^{-1} 3132,2962,2928,2856,1667,1620$, 1446, 1325, 1218, 1166, 1127, 1067, 1018, 823; ${ }^{1} \mathrm{H}$ NMR $\left(300 \mathrm{MHz}, \mathrm{CDCl}_{3}\right) \delta 1.55(\mathrm{~s}, 3 \mathrm{H}), 1.63(\mathrm{~s}, 3 \mathrm{H}), 1.69(\mathrm{~s}$, $3 \mathrm{H}), 1.90$ (quint, $2 \mathrm{H}, J 6.3 \mathrm{~Hz}$ ), $2.02(\mathrm{~s}, 4 \mathrm{H}), 2.76(\mathrm{t}, 2 \mathrm{H}$, $J 6.3 \mathrm{~Hz}), 3.41(\mathrm{t}, 2 \mathrm{H}, J 6.3 \mathrm{~Hz}), 3.90(\mathrm{~d}, 2 \mathrm{H}, J 6.9 \mathrm{~Hz})$, 5.05 (brs, 1H), 5.29 (t, 1H, J 6.9 Hz), 5.52 (s, 2H), 7.24 (s, 1H), $7.31(\mathrm{~d}, 2 \mathrm{H}, J 8.4 \mathrm{~Hz}), 7.58(\mathrm{~d}, 2 \mathrm{H}, J 8.4 \mathrm{~Hz})$; ${ }^{13} \mathrm{C} \mathrm{NMR}\left(75 \mathrm{MHz}, \mathrm{CDCl}_{3}\right) \delta 17.6,22.3,23.4,25.6,26.6$, 29.3, 32.2, 53.2, 67.0, 69.1, 120.9, 121.8, 123.8, 124.8 (q, $\left.J_{\mathrm{C}-\mathrm{F}} 270.0 \mathrm{~Hz}\right), 126.0\left(\mathrm{q}, J_{\mathrm{C}-\mathrm{F}} 3.6 \mathrm{~Hz}\right), 128.0,130.8\left(\mathrm{q}, J_{\mathrm{C}-\mathrm{F}}\right.$ $32.4 \mathrm{~Hz}), 131.8,139.0,140.2$, 148.5; MS-EI, $\mathrm{m} / \mathrm{z}(\%): 421$ (0.2) $\mathrm{C}_{23} \mathrm{H}_{30} \mathrm{~F}_{3} \mathrm{~N}_{3} \mathrm{O}\left[\mathrm{M}^{+*}\right], 353$ (2), 286 (40), 268 (44), 241 (77), 212 (3), 159 (100), 140 (5), 121 (11), 109 (33), 93 (30), 80 (17), 69 (62), 53 (21), 41 (96).

(Z)-4-(3-((3,7-Dimethylocta-2,6-dien-1-yl)oxy)propyl)1-(4-methoxybenzyl)-1H-1,2,3-triazole (10h)

Compound 10h was obtained as a yellow oil in $76 \%$ yield (186 mg, $0.480 \mathrm{mmol}$ ) from compounds $3 \mathbf{h}(122 \mathrm{mg}$, $0.750 \mathrm{mmol})$ and $8(165 \mathrm{mg}, 0.750 \mathrm{mmol}) .25 \mathrm{mg}$ of unreacted compound $\mathbf{8}$ was also recovered. TLC: $\mathrm{Rf}=0.34$ (hexane/ethyl acetate/dichloromethane 3:1:3 v/v); IR (ATR) $\bar{v}_{\max } / \mathrm{cm}^{-1} 3131,3072,2959,2929,2854,1714,1667,1550$, 1514, 1445, 1375, 1249, 1178, 1102, 1034, 823, 783, 514; ${ }^{1} \mathrm{H}$ NMR (300 MHz, $\left.\mathrm{CDCl}_{3}\right) \delta 1.56$ (s, 3H), 1.63 (s, 3H), 1.70 (s, 3H), 1.88 (quint, $2 \mathrm{H}, J 6.3 \mathrm{~Hz}$ ), 2.03 (s, 4H), 2.72 (t, 2H, J 6.3 Hz), 3.40 (t, 2H, J $6.3 \mathrm{~Hz}$ ), 3.76 (s, 3H), 3.89 (d, $2 \mathrm{H}, J 6.9 \mathrm{~Hz}), 5.05$ (brs, 1H), $5.29(\mathrm{t}, 1 \mathrm{H}, J 6.9 \mathrm{~Hz})$, $5.38(\mathrm{~s}, 2 \mathrm{H}), 6.84(\mathrm{~d}, 2 \mathrm{H}, J 8.4 \mathrm{~Hz}), 7.15(\mathrm{~s}, 1 \mathrm{H}), 7.18(\mathrm{~d}$, $J$ 8.4 Hz); ${ }^{13} \mathrm{C}$ NMR $\left(75 \mathrm{MHz}, \mathrm{CDCl}_{3}\right) \delta 17.5,22.2,23.3$, 25.6, 26.6, 29.3, 32.1, 53.4, 55.2, 66.9, 69.1, 114.3, 120.4, 121.8, 123.7, 126.8, 129.4, 131.8, 140.1, 147.9, 159.7; MS-EI, $m / z(\%): 383(0.3) \mathrm{C}_{23} \mathrm{H}_{33} \mathrm{~N}_{3} \mathrm{O}_{2}\left[\mathrm{M}^{+}\right], 315$ (4), 262 (2), 248 (13), 231 (5), 203 (20), 121 (100), 110 (16), 93 (15), 77 (18), 69 (27), 53 (11), 41 (58).

(Z)-4-(3-((3,7-Dimethylocta-2,6-dien-1-yl)oxy)propyl)1-(4-nitrobenzyl)-1H-1,2,3-triazole (10i)

Compound 10i was obtained as a yellow oil in $71 \%$ yield (200 mg, $0.500 \mathrm{mmol}$ ) from compounds $3 \mathbf{i}(134 \mathrm{mg}$, $0.750 \mathrm{mmol})$ and $\mathbf{8}(165 \mathrm{mg}, 0.750 \mathrm{mmol}) .20 \mathrm{mg}$ of unreacted compound $\mathbf{8}$ was also recovered. Yellow oil, TLC: $\mathrm{Rf}=0.26$ (hexane/ethyl acetate/dichloromethane $3: 1: 3 \mathrm{v} / \mathrm{v})$; IR (ATR) $\overline{\mathrm{v}}_{\max } / \mathrm{cm}^{-1} 3133,3078,2961,2926$, 2855, 1668, 1606, 1522, 1446, 1346, 1218, 1105, 1046, $858,804,734 ;{ }^{1} \mathrm{H}$ NMR (300 MHz, $\left.\mathrm{CDCl}_{3}\right) \delta 1.56(\mathrm{~s}, 3 \mathrm{H})$, $1.64(\mathrm{~s}, 3 \mathrm{H}), 1.71$ (s, 3H), 1.92 (quint, $2 \mathrm{H}, J 6.3 \mathrm{~Hz}$ ), 2.03 (s, 4H), 2.78 (t, 2H, J $6.3 \mathrm{~Hz}), 3.43$ (t, 2H, J $6.3 \mathrm{~Hz}), 3.91$ (d, $2 \mathrm{H}, J 6.9 \mathrm{~Hz}), 5.05$ (brs, 1H), $5.30(\mathrm{t}, 1 \mathrm{H}, J 6.9 \mathrm{~Hz})$,
5.59 (s, 2H), $7.28(\mathrm{~s}, 1 \mathrm{H}), 7.35(\mathrm{~d}, 2 \mathrm{H}, J 8.4 \mathrm{~Hz}), 8.18$ (d, $2 \mathrm{H}, J 8.4 \mathrm{~Hz}) ;{ }^{13} \mathrm{C}$ NMR $\left(75 \mathrm{MHz}, \mathrm{CDCl}_{3}\right) \delta 17.6,22.4$, 23.5, 25.7, 26.7, 29.4, 32.2, 52.9, 67.0, 69.1, 121.0, 121.8, $123.8,124.2,128.4,131.9,140.3,142.1,147.9,148.8$; MSEI, $m / z(\%): 398(0.2) \mathrm{C}_{22} \mathrm{H}_{30} \mathrm{~N}_{4} \mathrm{O}_{3}\left[\mathrm{M}^{+*}\right], 330(0.3), 263(8)$, 245 (12), 218 (22), 199 (5), 136 (10), 121 (4), 106 (19), 89 (15), 78 (34), 69 (58), 53 (18), 41 (100).

(Z)-1-(3,4-Difluorobenzyl)-4-(3-((3,7-dimethylocta-2,6-dien1-yl)oxy)propyl)-1H-1,2,3-triazole (10j)

Compound $10 \mathbf{j}$ was obtained as a yellow oil in $96 \%$ yield ( $276 \mathrm{mg}, 0.710 \mathrm{mmol}$ ) from compounds $\mathbf{3 j}$ ( $126.8 \mathrm{mg}$, $0.750 \mathrm{mmol})$ and $8(165 \mathrm{mg}, 0.750 \mathrm{mmol}) .5 \mathrm{mg}$ of unreacted compound $\mathbf{8}$ was also recovered. TLC: $\mathrm{Rf}=0.35$ (hexane/ethyl acetate/dichloromethane 3:1:3 v/v); IR (ATR) $\bar{v}_{\max } / \mathrm{cm}^{-1} 3133,2960,2926,2856,1715,1668,1611$, 1519, 1439, 1376, 1286, 1214, 1115, 1047, 782, 574, 524, 452; ${ }^{1} \mathrm{H}$ NMR (300 MHz, $\left.\mathrm{CDCl}_{3}\right) \delta 1.56(\mathrm{~s}, 3 \mathrm{H}), 1.63$ (s, $3 \mathrm{H}), 1.70(\mathrm{~s}, 3 \mathrm{H}), 1.91$ (quint, $2 \mathrm{H}, J 6.3 \mathrm{~Hz}$ ), $2.03(\mathrm{~s}, 4 \mathrm{H}$ ), 2.76 (t, 2H, J $6.3 \mathrm{~Hz}), 3.41$ (t, 2H, J $6.3 \mathrm{~Hz}), 3.90$ (d, 2H, $J 6.9 \mathrm{~Hz}$ ), 5.05 (brs, 1H), 5.30 (t, 1H, J 6.9 Hz), 5.42 (s, 2H), 6.99-7.17 (m, 3H), 7.23 (s, 1H); ${ }^{13} \mathrm{C}$ NMR (75 MHz, $\left.\mathrm{CDCl}_{3}\right) \delta 17.6,22.3,23.4,25.6,26.6,29.3,32.2,52.8$, 67.0, 69.1, 116.9-117.1 (m), 117.7-118.0 (m), 120.8, 121.8, $123.8,123.9-124.0(\mathrm{~m}), 131.9,140.2,148.5,148.6-148.9$ (m), 151.9-152.0 (m), 152.1-152.2 (m); MS-EI, $\mathrm{m} / \mathrm{z}(\%)$ : 389 (0.20) $\mathrm{C}_{22} \mathrm{H}_{29} \mathrm{~F}_{2} \mathrm{~N}_{3} \mathrm{O}\left[\mathrm{M}^{+*}\right], 321$ (3), 262 (5), 254 (50), 236 (39), 209 (74), 180 (3), 127 (100), 93 (36), 80 (20), 69 (58), 53 (22), 41 (80).

(Z)-1-(2,5-Dichlorobenzyl)-4-(3-((3,7-dimethylocta-2,6-dien1-yl)oxy)propyl)-1H-1,2,3-triazole (10k)

Compound 10k was obtained as a yellow oil in $70 \%$ yield (174 mg, $0.410 \mathrm{mmol}$ ) from compounds $3 \mathbf{k}(152 \mathrm{mg}$, $0.750 \mathrm{mmol})$ and $8(165 \mathrm{mg}, 0.750 \mathrm{mmol}) .35 \mathrm{mg}$ of unreacted compound $\mathbf{8}$ was also recovered. TLC: $\mathrm{Rf}=0.51$ (hexane/ethyl acetate/dichloromethane 3:1:3 v/v); IR (ATR) $\bar{v}_{\max } / \mathrm{cm}^{-1} 3134,3071,2959,2926,2855,1722$, 1667, 1552, 1464, 1376, 1272, 1219, 1099, 1045, 815, 522; ${ }^{1} \mathrm{H}$ NMR (300 MHz, $\left.\mathrm{CDCl}_{3}\right) \delta 1.57$ (s, 3H), 1.65 (s, 3H), 1.71 (s, 3H), 1.93 (quint, $2 \mathrm{H}, J 6.3 \mathrm{~Hz}$ ), 2.04 (s, 4H), 2.79 (t, 2H, J $6.3 \mathrm{~Hz}$ ), 3.43 (t, 2H, J 6.3 Hz), 3.92 (d, $2 \mathrm{H}, J 6.9 \mathrm{~Hz}$ ), 5.08 (brs, 1H), 5.32 (t, 1H, J $6.9 \mathrm{~Hz}), 5.56$ (s, 2H), $7.06(\mathrm{~d}, 1 \mathrm{H}, J 2.1 \mathrm{~Hz}), 7.24\left(\mathrm{dd}, 1 \mathrm{H}, J_{1} 8.4\right.$ and $\left.J_{2} 2.1 \mathrm{~Hz}\right), 7.32(\mathrm{~s}, 1 \mathrm{H}), 7.34(\mathrm{~d}, 1 \mathrm{H}, J 8.4 \mathrm{~Hz}) ;{ }^{13} \mathrm{C} \mathrm{NMR}$ $\left(75 \mathrm{MHz}, \mathrm{CDCl}_{3}\right) \delta 17.6,22.4,23.5,25.7,26.7,29.4,32.2$, 50.7, 67.0, 69.1, 121.2, 121.9, 123.8, 129.8, 130.0, 130.9, 131.3, 131.9, 133.5, 134.4, 140.2, 148.3; MS-EI, $\mathrm{m} / \mathrm{z}(\%)$ : $422(0.1) \mathrm{C}_{22} \mathrm{H}_{29} \mathrm{Cl}_{2} \mathrm{~N}_{3} \mathrm{O}\left[\mathrm{M}^{+*}\right], 353$ (1), 286 (11), 268 (13), 241 (38), 204 (4), 159 (47), 123 (13), 93 (15), 69 (58), 53 (15), 41 (100). 
Cell culture

Human leukemia cell lines HL60 (acute myelogenous leukemia, AML), Nalm6 (B-cell acute lymphoblastic leukemia, ALL-B), and Jurkat (T-cell acute lymphoblastic leukemia, ALL-T) were kindly provided by Dr Jose Andrés Yunes (Centro Infantil Boldrini, Campinas, São Paulo, Brazil). Mouse NIH3T3 and B16F10 cells were kindly provided by Dr Anésia Aparecida dos Santos (Departamento de Biologia Geral, Universidade Federal de Viçosa, Minas Gerais, Brazil). Human embryonic kidney HEK293 cell was kindly provided by Dr Jörg Kobarg (Instituto de Biologia, Universidade Estadual de Campinas, Campinas, São Paulo, Brazil). Cell lines were grown in Roswell Park Memorial Institute (RPMI)-1640 medium (Sigma, Darmstadt, Gemany) supplemented with $10 \%$ (v/v) fetal bovine serum (FBS) (LGC Biotecnologia, Cotia, São Paulo, Brazil), $100 \mathrm{~g} \mathrm{~mL}^{-1}$ streptomycin, and 100 units per $\mathrm{mL}$ penicillin at $\mathrm{pH} 7.2$ and $37^{\circ} \mathrm{C}$ under $5 \%$ $\mathrm{CO}_{2}$ atmosphere.

Cell viability assay

HL60, Nalm6, and Jurkat cells $\left(7 \times 10^{4}\right.$ cells well $\left.^{-1}\right)$, B16F10, NIH3T3, and HEK293 cells $\left(10^{4}\right.$ cells well $\left.^{-1}\right)$ were seeded in 96-well plates. Each well contained $100 \mu \mathrm{L}$ of complete RPMI medium and $100 \mu \mathrm{L}$ of each compound solution at different concentrations. Cytarabine, vincristine sulfate, and etoposide (Sigma-Aldrich, Darmstadt, Gemany) were used as positive controls. The compounds were diluted in RPMI medium with $10 \%$ FBS and $0.4 \%$ DMSO (v/v, Sigma, Darmstadt, Gemany). After 48 h of culture, 3-(4,5-dimethylthiazol-2-yl)-2,5-diphenyltetrazolium bromide (MTT, 5 mg mL-1, Sigma, Darmstadt, Gemany) was added to the wells. After $3 \mathrm{~h}$ at $37^{\circ} \mathrm{C}$, the MTT solution was removed and it was added $100 \mu \mathrm{L}$ well ${ }^{-1}$ of DMSO to solubilize the formazan. Absorbance was measured at $540 \mathrm{~nm}$ in a microplate reader (SpectraMax M5, Molecular Devices, San Jose, USA).

\section{Trypan blue exclusion assay}

Trypan blue exclusion assays were performed as previously described. ${ }^{30}$ Jurkat cells were seeded at the density of $10^{4}$ cells per well in 96-well plates. The compound 9f was added at 25 or $50 \mu \mathrm{mol} \mathrm{L}{ }^{-1}$, and DMSO $(0.4 \% \mathrm{v} / \mathrm{v})$ or RPMI medium were used as control. The effect of these treatments on cell growth were determined by trypan blue (Invitrogen, Carlsbad, USA) dye exclusion. After 24, 48, and 72 h, a hemocytometer was loaded with cells to obtain the viable cell count.

\section{Wound healing assay}

B16F10 cells were seeded on 24-well plates and grown for 24 h to $80-90 \%$ confluence. Cells were washed twice with phosphate-buffered saline (PBS) and the monolayers were scraped with a micropipette tip to create a uniform scratch. After that, cells were washed with PBS again to remove the detached cells. Then, compound 9 f was added in the RPMI medium at different concentrations $(50,75$ and $\left.100 \mu \mathrm{mol} \mathrm{L}{ }^{-1}\right)$. DMSO-vehicle treatment $(0.4 \% \mathrm{v} / \mathrm{v})$ was used as control. Digital images of the wounded monolayers were obtained by photomicroscope EVOS fl (Life Technologies, Cotia, São Paulo, Brazil) at 0, 12, 24, and $48 \mathrm{~h}$. The unfilled scratched zones were quantified by Java's Image J software. ${ }^{31}$

\section{Statistical analysis}

All numeric data were obtained from three independent experiments and are shown as means \pm standard deviation. Analyses were performed using Microsoft Excel (Microsoft Office Software) and GraphPad Prism. ${ }^{32}$ Statistical analyses were conducted by one-way analysis of variance (ANOVA) followed by Tukey's test. $p<0.05$ was considered significant.

Calculation of physicochemical and pharmacokinetic properties

SMILES representations for compounds $9 \mathbf{a}-\mathbf{k}$ and 10a-k were obtained from 2D structures drawn in MarvinSketch. ${ }^{33}$ Ligands were then prepared with the LigPrep software, ${ }^{34}$ and relevant protonation states at $\mathrm{pH} 7.0$ were calculated with Epik. Forty four physicochemical and pharmacokinetic properties were calculated by the normal mode of QikProp. ${ }^{35}$

\section{D-QSAR study}

Three-dimensional structures were built in HyperChem 7 (Hyper Co.), using as reference the Nerol structure retrieved from the ZINC Database (code 12405252). ${ }^{36}$ All geometries were initially optimized by molecular mechanics (MM+), in cycles alternated with molecular dynamic studies (1 ps, $300 \mathrm{~K}$ ), until the energy obtained in $\mathrm{MM}+$ no longer varied, indicating a possible minimum energy structure. Next, these obtained structures were optimized by quantum mechanics, initially at AM1 semi-empirical level, in the same software. The geometries were exported to the software Gaussian $09^{37}$ and new optimizations were carried out at 
Hartree-Fock level (HF/6-31Gd,p) and, in the last step, by density functional theory (DFT) (B3LYP/Def2TZVPP). The B3LYP functional was chosen because it leads to quite satisfactory results for the analysis of geometries and energies. ${ }^{38,39}$ Besides, the balanced basis set Def2-TZVPP was chosen because it produces similar errors along all the elements from the periodic table. ${ }^{40}$

Using the optimized geometry, 3D descriptors were calculated in Pentacle software. ${ }^{41}$ The GRIND approach that calculates 3D maps of interaction energies between the molecule and chemical probes (GRIND based molecular interaction fields, MIFs), in an alignment-independent procedure, was used..$^{42}$ The molecular probes involved were: DRY, representing hydrophobic interactions, $\mathrm{O}$ ( $\mathrm{sp}^{2}$ carbonyl oxygen) as a hydrogen bond acceptor, N1 as hydrogen bond donor (NH like amide) and TIP that represents the shape of the molecule in terms of steric hot spots. The maximum field intensity at relative distances (in angstroms) was computed using the AMANDA algorithm and the pre-filtered nodes were encoded using the MACC2 algorithm.

Experimental biological activities were converted into their corresponding $\mathrm{pIC}_{50}$ (or $-\log \mathrm{IC}_{50}$, where $\mathrm{IC}_{50}$ is the concentration in mol L $\mathrm{L}^{-1}$ that inhibited cell proliferation by $50 \%$ when compared to untreated controls). A total of 629 GRIND descriptors were calculated for nerol and derivatives. The data generated was organized in a data matrix $\mathbf{X}(\mathrm{N} \times 629)$, where $\mathrm{N}$ is the number of active compounds. Four quantitative regression models, based on the experimental biological activities measured against cancer cell lines, were built.

Feature selection was initially carried out by using the fractional factorial design method (FDD), also available in Pentacle. ${ }^{41}$ The following step was to eliminate those descriptors that had the absolute value of Pearson's correlation coefficient $(|\mathrm{r}|)$ with biological activity lower than 0.2. Finally, the ordered predictors selection algorithm (OPS) ${ }^{43}$ implemented in the public domain QSAR modeling software, ${ }^{44}$ was used to define the final set of descriptors to be kept in the models. Descriptors and biological activities were autoscaled (column wise mean-centered and scaled to unity variance) prior to chemometric analysis.

Quantitative models were built using the partial least squares regression method (PLS) implemented in QSAR modeling software. ${ }^{44}$ The quality of the models was assessed based on their coefficient of determination $\left(\mathrm{R}^{2}\right)$ and the root mean square error of calibration (RMSEC), the $F$-ratio test with $95 \%$ confidence interval $(F, \alpha=0.05)$, the coefficient of determination in the leave-one-out (LOO) cross-validation $\left(\mathrm{Q}_{\mathrm{LOO}}^{2}\right)$, and the root mean square error of cross-validation (RMSECV). The robustness was tested by the leave- $\mathrm{N}$-out ( $\mathrm{LNO}$ ) cross validation test, where $\mathrm{N}=1$ to 5 for all models, and each $\mathrm{N}$ was repeated six times (in each replicate the rows from the data matrix were randomized). For a robust model, it is expected an average value of coefficients of determination obtained in each step of the LNO cross-validation (or average $\mathrm{Q}_{\mathrm{LNO}}^{2}$ ) close to $\mathrm{Q}_{\mathrm{LOO}}^{2}{ }^{45}$ Finally, the $\mathbf{y}$-randomization test was applied to confirm that the models did not suffer from chance correlation. For this test, the vector $\mathbf{y}$ (containing the biological activities) was scrambled 40 times, in accordance with the approach suggested by Eriksson et al. ${ }^{46}$ The selected descriptors that were included in the final models were interpreted in order to have some insight on which properties (structural, MIF, physiochemical and pharmacokinetic) could be related to the cytotoxic activities. Besides, molecular graphics of the selected descriptors and hierarchical cluster analysis (HCA) in Pirouette software ${ }^{47}$ were compared to explore some relationship among the cell lines.

\section{Results and Discussion}

\section{Synthesis of nerol derivatives}

The steps involved in the synthesis of nerol derivatives $\mathbf{9 a}-\mathbf{k}$ and $\mathbf{1 0 a}-\mathbf{k}$, containing a triazole moiety, are summarized in Scheme 1.

The alkylation of nerol (6) with propargyl bromide and pent-4-yn-1-yl methanesulfonate (5) gave alkylated compounds $\mathbf{7}$ and $\mathbf{8}$, respectively. Azides $\mathbf{3}$ were prepared from eleven benzylic alcohols. ${ }^{29}$ The CuAAC, also known as click reaction, between azides $\mathbf{3}$ and compounds $\mathbf{7}$ and $\mathbf{8}$ yielded the corresponding nerol derivatives with 1,2,3-triazole appendages. The reaction conditions used in the CuAAC were similar to those that we have utilized for the preparation of other 1,2,3-triazoles. ${ }^{16,48}$ As previously described, the main goal of the present investigation was to assess the cytotoxic potential of nerol 1,2,3-triazolic derivatives. As a consequence, an optimization of the reaction conditions was not carried out and some triazolic products were obtained with moderate yields. Moreover, in some click reactions it was recovered unreacted alkyne starting material. Compound structures were confirmed based on IR and NMR $\left({ }^{1} \mathrm{H}\right.$ and $\left.{ }^{13} \mathrm{C}\right)$ spectroscopies. The ${ }^{1} \mathrm{H}$ NMR spectra of nerol derivatives exhibited signals for the aryl group attached to triazole ring within 6.17-8.22 ppm range. In addition, the hydrogen of the heterocyclic ring was observed at 7.18-7.57 ppm. Once synthesized, compounds 9a-k and 10a-k as well as compounds $\mathbf{7}$ and $\mathbf{8}$ were biologically evaluated with respect to their cytotoxicity. It should be mentioned that it 
was varied the pattern of substitution of the benzyl ring as well as the length of the aliphatic chain in order to verify the influence of these factors on the biological activity.

\section{Cytotoxic activity}

The HL60 cell line commonly responds to potential antitumor agents with high sensitivity. ${ }^{49}$ Thus, this cell line was chosen to initially select nerol derivatives with potential cytotoxic effect. With exception of nerol (6) and compound $\mathbf{8}$, the remaining substances were able to reduce cell viability at $100 \mu \mathrm{mol} \mathrm{L} \mathrm{L}^{-1}$, the highest concentration evaluated (Figure 2). The most cytotoxic compounds identified were $9 \mathbf{e}, \mathbf{9 f}$, and $\mathbf{1 0 e}$, which reduced the HL60 viability to approximately $20 \%$ at $25 \mu \mathrm{mol} \mathrm{L}^{-1}$. This finding is in close agreement with previous work, which have already reported cytotoxic activity for some terpenoids and other compounds related to nerol. ${ }^{11,50}$ Cytarabine (Ara-C), vincristine (VCR), and etoposide (VP-16), components of many multi-drug pediatric and adult leukemia treatments were used as positive control, ${ }^{51}$ which decreased cell viability to $31.4,27.7$, and $11.1 \%$, respectively (Figure 2).

Compounds presenting cytotoxic activity against HL60 were selected for the determination of half maximal inhibitory concentration $\left(\mathrm{IC}_{50}\right)$ against other leukemias (Nalm6 and Jurkat) as well as melanoma (B16F10) and embryonic non-tumor cells (NIH3T3). The selected derivatives presented $\mathrm{IC}_{50}$ ranging from 9.84 to
84.2 $\mu$ mol L-1 against HL60, from 36.0 to $98.7 \mu \mathrm{mol} \mathrm{L}-1$ against Nalm6 and from 30.9 to $84.4 \mu \mathrm{mol} \mathrm{L}^{-1}$ against Jurkat (Table 1). Melanoma B16F10 cells were less sensitive, but four compounds (9d, 9f, 10f, and 10i) present moderate activity presenting $\mathrm{IC}_{50}$ values ranging from 74.7 to $98.2 \mu \mathrm{mol} \mathrm{L}-1$.

Among all evaluated substances, the derivative $9 f$ was, overall, the most active, as the determined $\mathrm{IC}_{50}$ values against HL60, Nalm6, Jurkat, and B16F10 were, respectively, $12.3,38.5,30.9$, and $74.7 \mu \mathrm{mol} \mathrm{L} \mathrm{L}^{-1}$. In addition, most of the derivatives showed lower cytotoxicity against the nontumor cell NIH3T3. Nine out of twenty-three evaluated compounds did not present any cytotoxic activity against this cell lineage, and another nine nerol derivatives presented $\mathrm{IC}_{50}$ values higher than $100 \mu \mathrm{mol} \mathrm{L} \mathrm{L}^{-1}$ (Table 1). Also, the most cytotoxic 9f compound showed some cytotoxicity $\left(\mathrm{IC}_{50} 37.33 \mu \mathrm{mol} \mathrm{L}{ }^{-1}\right)$ against the human non-cancerous cell line HEK293, but still lower than leukemic cells such as HL60 and Jurkat (see Supplementary Information).

\section{Effects of compound $9 f$ on cancer cell viability and migration}

The most active compound 9 f was used in additional experiments in order to gain insights on how it might acts in tumor cells. In the trypan blue exclusion assays, derivative 9f significantly impaired viability of Jurkat cells in a timeand concentration-dependent manner (Figure 3). This compound inhibited up to $70 \%$ of cell growth after $72 \mathrm{~h}$ in comparison to controls (DMSO and RPMI), suggesting

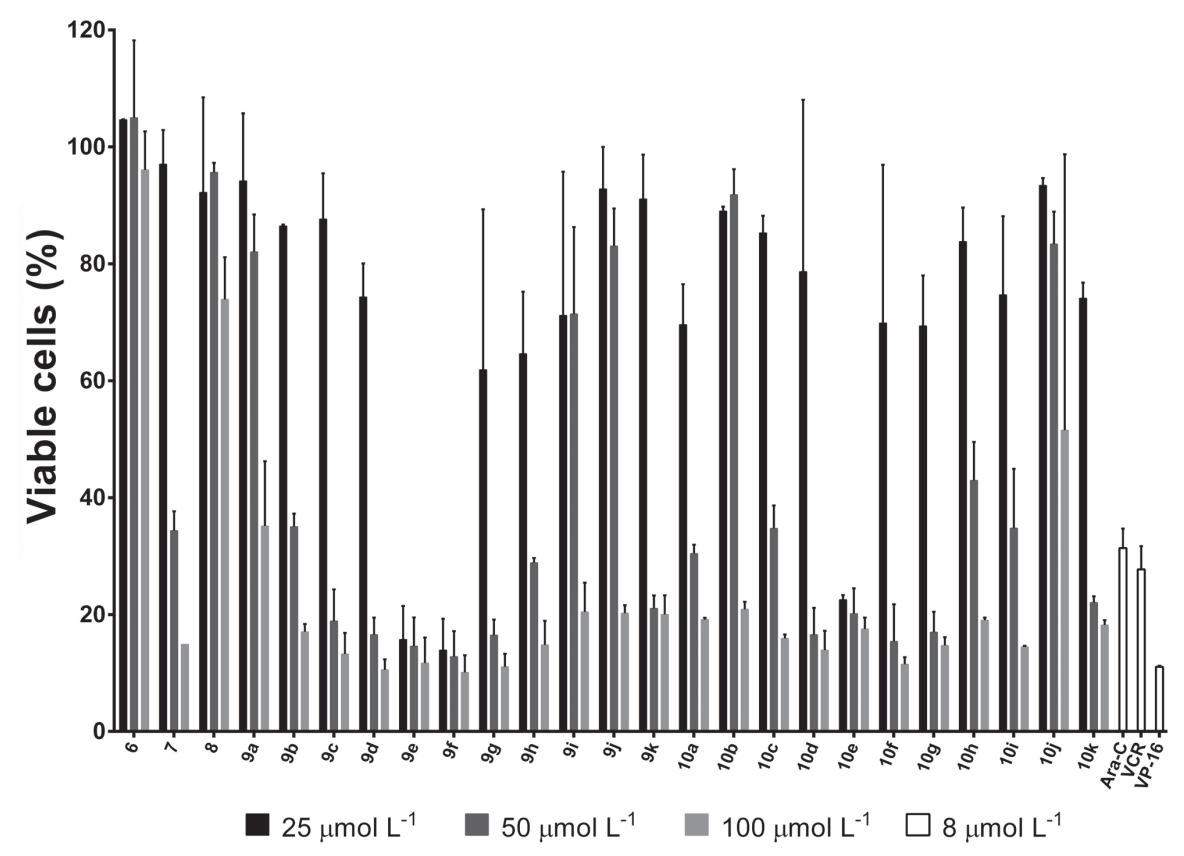

Figure 2. The effect of nerol and its derivatives on leukemia cell viability. HL60 cells were treated with different concentrations $\left(25,50\right.$ and $\left.100 \mu m o l L^{-1}\right)$ of nerol and its derivatives for $48 \mathrm{~h}$. Cytarabine (Ara-C), vincristine (VCR), and etoposide (VP-16) $\left(8 \mu \mathrm{mol} \mathrm{L}^{-1}\right)$ were used as positive control. Cell viability was determined using the MTT assay. The percentage of inhibition was calculated considering the cells treated with the vehicle (DMSO). 
Table 1. Half-maximal inhibitory concentration $\left(\mathrm{IC}_{50}\right)$ values for nerol derivatives. HL60, Nalm6 and Jurkat leukemic cells, B16F10 metastatic melanoma and NIH3T3 mouse embryonic cell line were treated with increasing concentrations $\left(0-200 \mu \mathrm{mol} \mathrm{L}^{-1}\right)$ of each compound for $48 \mathrm{~h}$. Cell viability was determined using the MTT assay. $\mathrm{IC}_{50}$ values are expressed as the means \pm standard deviation of three independent experiments

\begin{tabular}{|c|c|c|c|c|c|}
\hline \multirow[b]{2}{*}{ Compound } & \multicolumn{5}{|c|}{$\mathrm{IC}_{50} /(\mu \mathrm{mol} \mathrm{L}-1)$} \\
\hline & $\begin{array}{l}\text { HL60 } \\
\text { (AML) }\end{array}$ & $\begin{array}{c}\text { Nalm6 } \\
\text { (ALL-B) }\end{array}$ & $\begin{array}{c}\text { Jurkat } \\
\text { (ALL-T) } \\
\end{array}$ & B16F10 & NIH3T3 \\
\hline 7 & $41.7 \pm 2.94$ & $91.1 \pm 7.13$ & $80.2 \pm 2.90$ & $149.7 \pm 12.3$ & NA \\
\hline $9 a$ & $75.7 \pm 10.0$ & $98.7 \pm 1.89$ & NA & NA & NA \\
\hline $9 \mathrm{~b}$ & $44.9 \pm 2.97$ & $42.8 \pm 2.28$ & $84.4 \pm 31.0$ & NA & $101.0 \pm 0.88$ \\
\hline $9 \mathrm{c}$ & $33.1 \pm 2.58$ & $78.2 \pm 15.8$ & $55.4 \pm 3.33$ & $154.7 \pm 1.38$ & $110.6 \pm 4.68$ \\
\hline 9d & $36.4 \pm 3.51$ & $52.3 \pm 0.11$ & $61.6 \pm 2.51$ & $97.2 \pm 2.28$ & $113.3 \pm 16.6$ \\
\hline $9 e$ & $26.0 \pm 1.56$ & $39.9 \pm 4.14$ & $41.6 \pm 0.39$ & $144.5 \pm 2.49$ & $65.5 \pm 4.69$ \\
\hline $9 f$ & $12.3 \pm 4.33$ & $38.5 \pm 0.39$ & $30.9 \pm 18.8$ & $74.7 \pm 7.26$ & $58.2 \pm 2.21$ \\
\hline $9 \mathrm{~g}$ & $42.3 \pm 1.03$ & $61.0 \pm 0.66$ & $52.4 \pm 3.55$ & $124.4 \pm 5.4$ & $115.7 \pm 15.5$ \\
\hline $9 h$ & $57.9 \pm 6.84$ & NA & $75.1 \pm 20.9$ & NA & $102.4 \pm 0.45$ \\
\hline $9 \mathrm{i}$ & $59.2 \pm 4.86$ & $65.0 \pm 2.85$ & $69.5 \pm 7.09$ & $147.4 \pm 1.22$ & $102.5 \pm 1.09$ \\
\hline $9 \mathbf{j}$ & $84.2 \pm 14.8$ & $96.2 \pm 5.26$ & NA & NA & NA \\
\hline $9 k$ & $36.6 \pm 2.79$ & $50.6 \pm 4.96$ & $51.6 \pm 2.65$ & NA & NA \\
\hline $10 \mathrm{a}$ & $52.5 \pm 4.11$ & $78.2 \pm 4.73$ & NA & NA & NA \\
\hline $10 \mathrm{~b}$ & $66.4 \pm 6.97$ & NA & NA & NA & NA \\
\hline $10 \mathrm{c}$ & $45.3 \pm 9.58$ & $53.0 \pm 5.89$ & $65.1 \pm 4.91$ & $125.3 \pm 6.15$ & $117.6 \pm 10.2$ \\
\hline 10d & $42.3 \pm 4.60$ & $42.2 \pm 0.92$ & $52.3 \pm 6.55$ & $140.5 \pm 0.66$ & NA \\
\hline $10 e$ & $9.84 \pm 0.84$ & $53.2 \pm 1.60$ & $49.9 \pm 18.9$ & $131.7 \pm 0.73$ & $142.3 \pm 12.7$ \\
\hline $10 f$ & $40.3 \pm 2.19$ & $45.2 \pm 0.82$ & $73.6 \pm 31.5$ & $92.4 \pm 2.00$ & NA \\
\hline $10 \mathrm{~g}$ & $48.3 \pm 8.75$ & $45.9 \pm 6.63$ & $58.5 \pm 1.52$ & $134.1 \pm 4.58$ & $105.7 \pm 3.78$ \\
\hline $10 \mathrm{~h}$ & $45.2 \pm 0.26$ & NA & $78.7 \pm 2.30$ & NA & $84.8 \pm 13.2$ \\
\hline $10 \mathrm{i}$ & $44.5 \pm 0.49$ & $36.0 \pm 0.15$ & $37.1 \pm 16.3$ & $98.2 \pm 0.28$ & NA \\
\hline $10 \mathrm{j}$ & $63.0 \pm 10.6$ & $72.4 \pm 6.68$ & NA & $174.8 \pm 16.9$ & $97.2 \pm 16.8$ \\
\hline $10 \mathrm{k}$ & $32.5 \pm 1.70$ & $43.2 \pm 3.67$ & $51.9 \pm 9.96$ & $145.3 \pm 4.41$ & $84.2 \pm 8.20$ \\
\hline
\end{tabular}

AML: acute myelogenous leukemia; ALL-B: B-cell acute lymphoblastic leukemia; ALL-T: T-cell acute lymphoblastic leukemia; NA: not active.

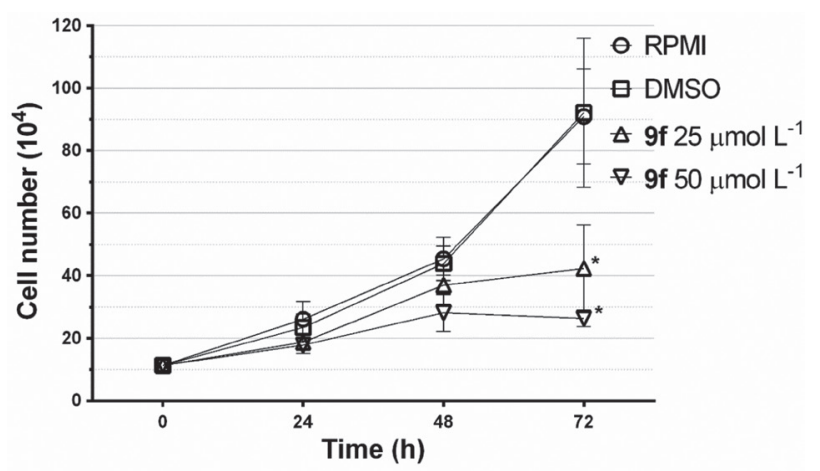

Figure 3. The effect of compound 9 f on cell viability over time. Jurkat cells were treated with 25 and $50 \mu \mathrm{mol} \mathrm{L}^{-1}$ of 9 f. Cells treated with vehicle (DMSO) and culture medium (RPMI) were used as control. Cell growth was determined with trypan blue exclusion at $0,24,48$, and $72 \mathrm{~h}$ after incubation. Data are shown as means \pm standard deviation of triplicate experiments $(* p<0.05)$.

that pathways affecting cell death and proliferation might be affected by the treatments.

Although melanoma B16F10 is commonly resistant to potential anticancer agents in cytotoxic assays, evaluation of other phenotypes would render additional information on the biological activity of compound 9 f. Thus, wound healing assays were carried out to evaluate the anti-migratory effect. Indeed, representative micrographies show that compound 9f efficiently inhibits B16F10 migration in comparison with DMSO in a time-dependent manner (Figure 4). These data suggests that adherent cells would be targeted in their migratory capacity by compound $\mathbf{9 f}$, potentially affecting the ability of colonization activity of these tumors.

\section{Analysis of estimated physicochemical and pharmacokinetic properties}

In addition to anti-cancer activity, it is important to consider several other properties relevant for drug development. Therefore, aiming to evaluate if compounds are suitable for further steps of lead optimization, a set of 44 properties were estimated for the compounds herein reported, employing the software QikProp. ${ }^{35}$

Especially for orally available drugs, most often compounds obey Lipinski's and Veber's rules..$^{52,53}$ According to Lipinski's rule of five, the following druglike physicochemical properties presents no more than one 

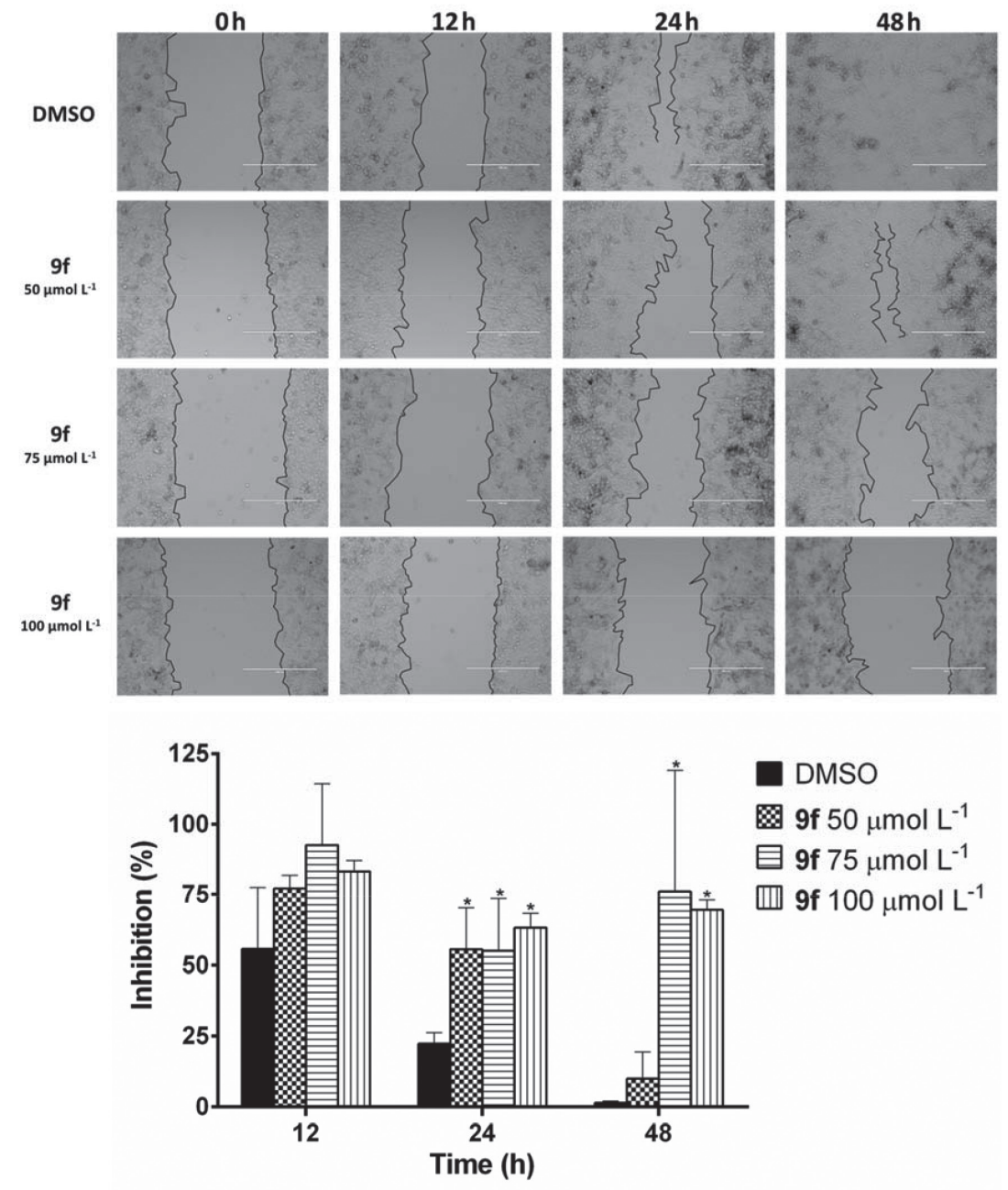

DMSO

冈 90 f $50 \mathrm{~mol} \mathrm{~L}^{-1}$ 目 9 f $75 \mu \mathrm{mol} \mathrm{L}^{-1}$ 四 9f $100 \mu \mathrm{mol} \mathrm{L}^{-1}$

Time (h)

Figure 4. The effect of derivative 9f on cell migration. B16F10 cells were treated with 50, 75, and $100 \mu \mathrm{mol} \mathrm{L}^{-1}$ of 9 f. Cells treated with vehicle (DMSO) and culture medium (RPMI) were used as control. (Top) Cell migration was determined by photographs of wound healing assay at $0,12,24$, and $48 \mathrm{~h}$ after incubation; (bottom) wound healing assay for percentage of inhibition of cell migration caused by 9 f in these three different concentrations. Error bars represent the means \pm standard deviation from triplicate experiment $(* p<0.05)$.

violation: molecular weight $\left(\leq 500 \mathrm{~g} \mathrm{~mol}^{-1}\right), \operatorname{clog} \mathrm{P}(\leq 5)$, number of hydrogen bond donors $(\leq 5)$ and number of hydrogen bond donors $(\leq 10)$. Besides, Veber's rules indicate that the number of rotatable bonds $(\leq 10)$ and total polar surface area (TPSA, $\leq 75 \AA^{3}$ ) are important as drug-like properties. Among the compounds studied here, $9 \mathbf{i}$ obeys all Lipinski's rules, while the remaining compounds violate one rule, $\operatorname{clog} \mathrm{P}$ (Table 2). Considering Veber's rules, eleven compounds or approximately half of the total have 10 or fewer rotatable bonds. Except for compound 10i, all other nerol derivatives present TPSA within the suggested limit. Therefore, overall the compounds herein investigated obey most physicochemical drug-like properties. The most promising compound in this study, derivative 9f, violates only the $\operatorname{cog} \mathrm{P} \operatorname{limit}(\mathrm{c} \log \mathrm{P}=6.4)$.

Several other properties which could be applicable for drug development (Table 3), such as the estimated affinity to HERG K+ channels (QPlogHERG), permeability through
Caco-2 (QPPCaco) and MDCK cells (QPPMDCK) and skin $\left(\mathrm{QPlog} \mathrm{K}_{\mathrm{p}}\right)$ and the number of predicted metabolic sites (No. metab), were also calculated. This analysis indicated properties which should be improved when optimizing this class of compounds, such as the affinity for HERG. Binding to $\mathrm{HERG} \mathrm{K}^{+}$channel is a common cause of cardiac toxicity, and therefore it is important to minimize interaction with this protein. Another aspect that deserves attention is the predicted number of metabolic sites, which is slightly higher than the range observed for $95 \%$ of drugs. While most drugs have at most 8 metabolic sites, compounds reported herein have 9 or 10 . To verify if this compromises the potential efficacy of these compounds, in vivo studies would be necessary.

On the other hand, the compounds described in this study are predicted to have very good cell permeability, which is an essential pharmacokinetic property. Except for compound 10i, all molecules were predicted to have 
Table 2. Calculated molecular properties for compounds $9 \mathbf{a}-\mathbf{k}$ and 10a-k. Estimated properties are considered relevant for drug-likeness according to the Lipinski's and Veber's rules

\begin{tabular}{|c|c|c|c|c|c|c|c|}
\hline Compound & $\begin{array}{c}\text { Molecular weight / } \\
\left(\mathrm{g} \mathrm{mol}^{-1}\right)\end{array}$ & $\operatorname{clog} \mathrm{P}$ & $\begin{array}{l}\text { No. H-bond } \\
\text { donors }\end{array}$ & $\begin{array}{l}\text { No. H-bond } \\
\text { acceptors }^{\mathrm{a}}\end{array}$ & $\begin{array}{l}\text { No. Lipinski's } \\
\text { rule violations }\end{array}$ & $\begin{array}{c}\text { No. of rotatable } \\
\text { bonds }\end{array}$ & TPSA / $\AA^{3}$ \\
\hline $9 \mathrm{a}$ & 325.5 & 5.3 & 0 & 4.2 & 1 & 9 & 40.9 \\
\hline $9 b$ & 343.4 & 5.5 & 0 & 4.2 & 1 & 9 & 40.9 \\
\hline $9 c$ & 359.9 & 5.8 & 0 & 4.2 & 1 & 9 & 40.8 \\
\hline 9d & 404.3 & 5.9 & 0 & 4.2 & 1 & 9 & 40.8 \\
\hline $9 e$ & 451.3 & 5.6 & 0 & 4.2 & 1 & 9 & 41.8 \\
\hline 9f & 409.5 & 6.4 & 0 & 4.2 & 1 & 10 & 48.6 \\
\hline $9 \mathrm{~g}$ & 393.5 & 6.3 & 0 & 4.2 & 1 & 9 & 40.8 \\
\hline $9 \mathrm{~h}$ & 355.5 & 5.3 & 0 & 4.95 & 1 & 10 & 49.1 \\
\hline $9 \mathrm{i}$ & 370.5 & 4.5 & 0 & 5.2 & 0 & 10 & 85.7 \\
\hline $9 j$ & 361.4 & 5.7 & 0 & 4.2 & 1 & 9 & 40.8 \\
\hline $9 \mathbf{k}$ & 394.3 & 6.2 & 0 & 4.2 & 1 & 9 & 40.5 \\
\hline $10 \mathrm{a}$ & 353.5 & 6.0 & 0 & 4.2 & 1 & 11 & 41.8 \\
\hline $10 \mathrm{~b}$ & 371.5 & 6.3 & 0 & 4.2 & 1 & 11 & 41.8 \\
\hline $10 \mathrm{c}$ & 388.0 & 6.6 & 0 & 4.2 & 1 & 11 & 41.7 \\
\hline 10d & 432.4 & 6.7 & 0 & 4.2 & 1 & 11 & 41.8 \\
\hline $10 \mathrm{e}$ & 497.4 & 6.7 & 0 & 4.2 & 1 & 11 & 41.8 \\
\hline $10 f$ & 437.5 & 7.2 & 0 & 4.2 & 1 & 12 & 49.5 \\
\hline $10 \mathrm{~g}$ & 421.5 & 7.1 & 0 & 4.2 & 1 & 11 & 41.8 \\
\hline $10 \mathrm{~h}$ & 383.5 & 6.1 & 0 & 4.95 & 1 & 12 & 50.1 \\
\hline $10 \mathrm{i}$ & 398.5 & 5.4 & 0 & 5.2 & 1 & 12 & 86.7 \\
\hline $10 \mathrm{j}$ & 389.5 & 6.5 & 0 & 4.2 & 1 & 11 & 41.8 \\
\hline $10 \mathrm{k}$ & 422.4 & 6.9 & 0 & 4.2 & 1 & 11 & 41.4 \\
\hline
\end{tabular}

${ }^{a}$ The number of hydrogen bond acceptors represents an average calculated based on a set of configurations, and therefore can be non-integer. TPSA: total polar surface area.

Table 3. Additional predicted pharmacokinetic properties for compounds $\mathbf{9 a}-\mathbf{k}$ and $\mathbf{1 0 a}-\mathbf{k}$

\begin{tabular}{|c|c|c|c|c|c|}
\hline Compound & QPlogHERG $^{\mathrm{a}}$ & $\mathrm{QPPCaco} /\left(\mathrm{nm} \mathrm{s}^{-1}\right)$ & $\mathrm{QPPMDCK}^{\mathrm{c}} /\left(\mathrm{nm} \mathrm{s}^{-1}\right)$ & $\mathrm{QP} \log \mathrm{K}_{\mathrm{p}}{ }^{\mathrm{d}}$ & No. metab \\
\hline $\begin{array}{l}\text { Range } \text { or reference } \text { values }\end{array}$ & $>-5$ & $>500$ & $>500$ & -8.0 to -1.0 & 1 to 8 \\
\hline $9 a$ & -6.4 & 3118 & 1691 & -0.75 & 9 \\
\hline $9 \mathrm{~b}$ & -6.2 & 3135 & 3079 & -8.79 & 9 \\
\hline $9 c$ & -6.3 & 3138 & 4204 & -9.12 & 9 \\
\hline 9d & -6.3 & 3146 & 4533 & -9.13 & 9 \\
\hline $9 e$ & -5.4 & 3229 & 5043 & -9.76 & 9 \\
\hline 9f & -6.0 & 3375 & 8602 & -8.11 & 10 \\
\hline $9 \mathrm{~g}$ & -6.3 & 3146 & 7452 & -9.77 & 9 \\
\hline $9 h$ & -5.8 & 3374 & 1842 & -7.89 & 10 \\
\hline $9 \mathrm{i}$ & -6.2 & 376 & 172 & -2.64 & 10 \\
\hline $9 \mathbf{j}$ & -6.1 & 3147 & 5241 & -9.94 & 9 \\
\hline $9 k$ & -6.1 & 3120 & 8121 & -1.04 & 9 \\
\hline $10 \mathrm{a}$ & -6.4 & 3492 & 1912 & -4.81 & 9 \\
\hline $10 \mathrm{~b}$ & -6.4 & 3497 & 3465 & -6.15 & 9 \\
\hline $10 \mathrm{c}$ & -6.6 & 3504 & 4737 & -6.47 & 9 \\
\hline 10d & -6.4 & 3497 & 5082 & -6.51 & 9 \\
\hline $10 \mathrm{e}$ & -6.5 & 3493 & 5491 & -6.54 & 9 \\
\hline $10 f$ & -6.5 & 3494 & 8930 & -6.06 & 10 \\
\hline $10 \mathrm{~g}$ & -6.4 & 3496. & 8351 & -7.16 & 9 \\
\hline $10 \mathrm{~h}$ & -6.4 & 3476 & 1902 & -5.87 & 10 \\
\hline $10 \mathrm{i}$ & -6.6 & 420 & 194 & -2.38 & 10 \\
\hline $10 \mathrm{j}$ & -6.3 & 3506 & 5889 & -7.32 & 9 \\
\hline $10 \mathrm{k}$ & -6.2 & 3533 & 9264 & -7.54 & 9 \\
\hline
\end{tabular}

${ }^{a}$ Predicted $\log \mathrm{IC}_{50}$ for $\mathrm{HERG} \mathrm{K}^{+}$blockage; ${ }^{\mathrm{b}}$ predicted apparent Caco- 2 cell permeability for non-active transport; ${ }^{\mathrm{c}}$ predicted apparent MDCK cell permeability for non-active transport; ${ }^{\mathrm{d}}$ predicted skin permeability, $\log \mathrm{K}_{\mathrm{p}}$; ${ }^{\mathrm{e}}$ number of likely metabolic sites; ${ }^{\mathrm{f}}$ range observed for $95 \%$ of known drugs. 
great permeability through Caco-2 cells, a model for the gut-blood barrier, and MDCK cells, which model the blood brain barrier. Additionally, fourteen of the twenty compounds reported have skin permeability within the range observed for $95 \%$ of known drugs.

Therefore, most of the evaluated parameters seemed favorable for drug development, principally for compound 9f. These data certainly would favor next optimization steps in order to obtain more selective and potent substances against cancer. Together with the cytotoxicity results against cancer cells lines, these findings enforce nerol derivatives containing triazolic portion as a new class of therapeutic agents to be exploited and optimized.

Correlation coefficient between each property included in Tables 2 and 3 and $-\operatorname{logIC}{ }_{50}$ was calculated to better understand their real influence in the measured activities. The results are shown in Table 4.

As expected, the embryonic non-tumor cells NH3T3 presents a completely different behavior, compared to others. Permeability through skin $\left(\mathrm{QPlog} \mathrm{K}_{\mathrm{p}}\right)$ is the only pharmacokinetic property that shows some correlation $(-0.46)$ with biological activity of this cell line. Lower values of $\mathrm{QPlog} \mathrm{K}_{\mathrm{p}}$ favor biological activity. Molecular weight shows a good correlation with activity of leukemia cells and metastatic melanoma $(0.71,0.55,0.60$ and 0.55 for HL60, Nalm6, Jurkat and B16F10 cells, respectively). In general, the higher the molecular weight better is the activity. The number of H-bond acceptors and donors, the number of rotatable bonds, TPSA and QPPCaco seems not to be significant to any of the biological activities measured. Low positive correlation was observed between $\operatorname{cog} \mathrm{P}$ and activities from HL60, Nalm6, and B16F10 cells. The same can be stated regarding QPPMDCK and activities from leukemia cells and metastatic melanoma. On the other hand, there exists a small negative correlation between QPlogHERG and activities from Jurkat (-0.31) and B16F10 $(-0.35)$. These negative correlations mean that biological activity increases as the QPlogHERG values decrease.

\section{D-QSAR analysis}

In order to gain information about the structure of nerol derivatives and their ability to inhibit cancer cell lines, QSAR analyses were carried out. Only those compounds that showed activity against leukemia and metastatic melanoma cells (HL60: $\mathrm{n}=23$; Jurkat: $\mathrm{n}=18$; Nalm6: $\mathrm{n}=20$; B16F10: $\mathrm{n}=15$ ) were considered. Even though the number of compounds is small for a QSAR study, it is assumed as reasonable in terms of the number of molecules for an exploratory analysis, and that is the reason why data splitting-based model validations (external validation, bootstrapping) were not applied. Variable selection was carried out in two steps: firstly using the FDD and then OPS algorithm implemented in QSAR modeling. Four regression models were obtained and the regression coefficients (autoscaled) together with the models performance are presented in Table 5.

The model basic statistics $\left(\mathrm{R}^{2}\right.$ and $\left.\mathrm{Q}^{2}\right)$ satisfies the minimal requirements for $\mathrm{QSAR}$ studies $\left(\mathrm{R}^{2}>0.6, \mathrm{Q}^{2}>0.5\right)$. Standard errors (RMSEC and RMSECV) are below $5 \%$ of the mean value for $\mathrm{pIC}_{50}$. The number of latent variables varied from 2 to 5 . The models were validated using a set of procedures suggested in the literature. The robustness of each optimized model was confirmed by leave-N-out crossvalidation (LNO). Mean values of $\mathrm{Q}_{\mathrm{LNO}}^{2}$ oscillate around $\mathrm{Q}^{2}$ Loo within a narrow range, whilst the respective standard deviations are small for up to five of the samples taken

Table 4. Correlation coefficient between biological activities $\left(-\log \mathrm{IC}_{50}\right)$ and molecular/pharmacokinetic properties ${ }^{\mathrm{a}}$

\begin{tabular}{lccccc}
\hline & HL60 & Nalm6 & Jurkat & B16F10 & NH3T3 \\
\hline Molecular weight & 0.71 & 0.55 & 0.60 & 0.55 & 0.15 \\
clogP & 0.33 & 0.29 & 0.15 & 0.32 & -0.04 \\
H-Bond acceptors & -0.21 & -0.23 & 0.08 & -0.02 & 0.07 \\
No. of rotatable bonds & 0.04 & 0.01 & -0.01 & 0.21 & -0.14 \\
TPSA & -0.10 & 0.13 & 0.24 & 0.28 & -0.02 \\
QPlogHERG & -0.20 & -0.03 & -0.31 & 0.35 & -0.01 \\
QPPCaco & 0.27 & -0.11 & 0.09 & 0.41 & 0.13 \\
QPPMDCK & 0.39 & 0.38 & -0.09 & -0.16 & 0.17 \\
QPlogK & -0.21 & -0.05 & 0.21 & 0.34 & -0.46 \\
Metab & 0.07 & -0.09 & 0.22 & \\
\hline
\end{tabular}

aThe number of H-bond donors was not included since all values were equal to zero. TPSA: total polar surface area; QPlogHERG: predicted logIC for HERG K ${ }^{+}$blockage; QPPCaco: predicted apparent Caco-2 cell permeability for non-active transport; QPPMDCK: predicted apparent MDCK cell permeability for non-active transport; $Q P \log K_{\mathrm{p}}$ : predicted skin permeability, $\log \mathrm{K}_{\mathrm{p}}$. 
Table 5. Regression models obtained for each cell line and their internal statistics

\begin{tabular}{|c|c|c|c|}
\hline Model & Cell line & Regression equation $^{\mathrm{a}}$ & Statistics \\
\hline 1 & HL60 & $\begin{array}{c}\mathrm{pIC}_{50}=-0.803 \times(16 \text { _DRY-DRY }) \\
+0.363 \times(57 \text { DRY-DRY }) \\
-0.192 \times\left(202 \_T I P-T I P\right) \\
-0.414 \times(207 \text { TIP-TIP }) \\
+0.390 \times(221 \text { TIP-TIP }) \\
+0.909 \times\left(326 \_D R Y-N 1\right) \\
+0.286 \times(362 \text { DRY-N1 }) \\
+0.429 \times(401 \text { DRRY-TIP })\end{array}$ & $\begin{array}{c}\mathrm{R}^{2}=0.938 ; \mathrm{RMSEC}=0.053 ; F=42.361 ; \\
\mathrm{Q}^{2}{ }_{\mathrm{LOO}}=0.853 ; \mathrm{RMSECV}=0.081 ; \\
\text { average } \mathrm{Q}_{\mathrm{LNO}}^{2}=0.799 ; \text { intercept } \mathrm{R}^{2}=0.244 ; \\
\text { intercept } \mathrm{Q}^{2}{ }_{\mathrm{LOO}}=-1.282 ; \\
\text { cumulated information: } 75.12 \% ; \\
\text { latent variables: } 5\end{array}$ \\
\hline 2 & Nalm6 & $\begin{array}{l}\mathrm{pIC}_{50}=+0.205 \times\left(208 \_\mathrm{TIP}-\mathrm{TIP}\right) \\
\quad+0.220 \times\left(238 \_\mathrm{TIP}-\mathrm{TIP}\right) \\
\quad+0.242 \times\left(334 \_\mathrm{DRY}-\mathrm{N} 1\right) \\
\quad+0.375 \times\left(362 \_\mathrm{DRY}-\mathrm{N} 1\right) \\
\quad-0.564 \times\left(386 \_\mathrm{DRY}-\mathrm{TIP}\right) \\
\quad+0.194 \times\left(597 \_\mathrm{N} 1-\mathrm{TIP}\right)\end{array}$ & $\begin{array}{c}\mathrm{R}^{2}=0.872 ; \mathrm{RMSEC}=0.049 ; F=57.906 ; \\
\mathrm{Q}_{\mathrm{LOO}}^{2}=0.774 ; \mathrm{RMSECV}=0.064 ; \\
\text { average } \mathrm{Q}_{\mathrm{LNO}}^{2}=0.767 ; \text { intercept } \mathrm{R}^{2}=0.167 ; \\
\text { intercept } \mathrm{Q}_{\mathrm{LOO}}^{2}=-0.813 ; \\
\text { cumulated information: } 62.05 \% ; \\
\text { latent variables: } 2\end{array}$ \\
\hline 3 & Jurkat & 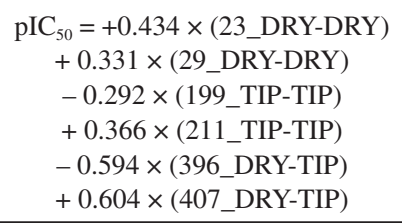 & $\begin{array}{c}\mathrm{R}^{2}=0.909 ; \mathrm{RMSEC}=0.035 ; \mathrm{Q}^{2}{ }_{\mathrm{LOO}}=0.804 ; \\
\mathrm{RMSECV}=0.052 ; F=32.464 ; \\
\text { average } \mathrm{Q}_{\mathrm{LNO}}^{2}=0.784 ; \text { intercept } \mathrm{R}^{2}=0.281 ; \\
\text { intercept } \mathrm{Q}_{\mathrm{LOO}}^{2}=-0.929 ; \\
\text { cumulated information: } 83.64 \% ; \\
\text { latent variables: } 4\end{array}$ \\
\hline 4 & B16F10 & $\begin{array}{c}\mathrm{pIC}_{50}=-0.184 \times\left(213 \_\mathrm{TIP}-\mathrm{TIP}\right) \\
+0.964 \times\left(362 \_\mathrm{DRY}-\mathrm{N} 1\right) \\
-0.149 \times\left(386 \_\mathrm{DRY}-\mathrm{TIP}\right) \\
-0.684 \times\left(415 \_\mathrm{DRY}-\mathrm{TIP}\right) \\
\quad+0.047 \times\left(573 \_\mathrm{N} 1-\mathrm{TIP}\right)\end{array}$ & $\begin{array}{c}\mathrm{R}^{2}=0.923 ; \mathrm{RMSEC}=0.027 ; \mathrm{Q}_{\mathrm{LOO}}^{2}=0.774 ; \\
\mathrm{RMSECV}=0.042 ; F=29.967 ; \\
\text { average } \mathrm{Q}_{\mathrm{LNO}}^{2}=0.796 ; \text { intercept } \mathrm{R}^{2}=0.200 ; \\
\text { intercept } \mathrm{Q}^{2} \text { Loo }=-1.361 ; \\
\text { cumulated information: } 92.17 \% ; \\
\text { latent variables: } 4\end{array}$ \\
\hline
\end{tabular}

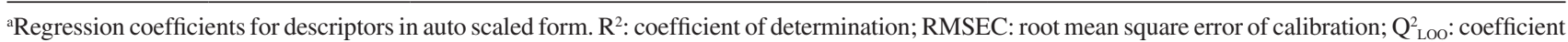
of determination in the leave-one-out (LOO) cross-validation; RMSECV: root mean square error of cross-validation; $F$ : $F$-ratio test with 95\% confidence interval; average $\mathrm{Q}_{\mathrm{LNO}}^{2}$ : average value of coefficients of determination obtained in each step of the $\mathrm{LNO}$ cross-validation.

out during the cross validation. The possibility of chance correlation was tested using $\mathbf{y}$-randomization analysis. Comparing randomized models (i.e., models with scrambled $\mathrm{pIC}_{50}$ values) with the final QSAR model, it is visible that the statistical parameters satisfy minimum criteria for the absence of chance correlation in all the four models. ${ }^{45,46}$

The number and type of MIF descriptors were different for each PLS regression models (Table 5). Comparing the four models based on their regression vector, it can be seen that $\mathrm{O}$ type probe (i.e., a carbonyl oxygen with hydrogen bond acceptor, HBA, properties) is not significant for cytotoxic activity. Being an HBA probe, it identifies hydrogen bond donor groups in the ligands (HBD) and this is in perfect agreement with the previous analysis on the physicochemical properties, since the molecules described do not have HBD groups (Table 4). From the eight selected descriptors in model 1 , the most important describes a hydrogen bond acceptor effect in the molecules combined to hydrophobic interaction (DRY-N1). One should have in mind that the N1 probe (which is HBD) identifies HBA groups in the ligands. The second most important descriptor has purely hydrophobic features (DRY-DRY). In this model, descriptors with combination of hydrophobic- steric (DRY-TIP) and purely steric descriptors (TIP-TIP) are also important. Regression model 2 is based on six MIF descriptors, where hydrophobic-steric (DRY-TIP) is the most significant feature. Pure hydrophobic effects (DRY-DRY) do not seem to be significant for model 2. The hydrogen bond acceptor-hydrophobic (DRY-N1) feature appears in other descriptors selected for this model. On the other hand, no N1 fields were selected in model 3, only DRY-DRY, TIP-TIP and DRY-TIP features appear to be important in this model. But in model 4, the DRY-N1 descriptor is the most important. As for model 2, pure hydrophobic effects (DRY-DRY) do not seem to be significant.

Models 1 and 3 present physicochemical information similar to each other because they present mainly purely hydrophobic (DRY-DRY), purely steric (TIP-TIP), and combination of both (DRY-TIP) features. The pairs of descriptors 199_TIP-TIP (HL60)/202_TIP-TIP (Jurkat), and 221_TIP-TIP (HL60)/211_TIP-TIP (Jurkat) highlight this similarity, which can be visualized in Figure 5 when each pair occupies equivalent regions in space. On the other hand, models 2 and 4 have two pairs of descriptors that are common, 362_DRY-N1 and 386_DRY-TIP. 
Despite their relative contribution in each equation is different, in both of them the signal is the same, positive for 362_DRY-N1 and negative for 386_DRY-TIP. Furthermore, as can be seen in Figure 6, both descriptors have the same behaviors, occupying similar regions in space, as would be expected in such situation.

These results suggest that the activities described may be occurring by similar mechanisms of action for the HL60 and Jurkat cell lines, and the same for Nalm6 and B16F10 cell lines. For a better understanding, an HCA based on the original $\mathrm{IC}_{50}$ values of all the compounds in the data set, was carried out. This approach was used to compare the behavior of the compounds as a whole, not just between the active compounds of each subset. For this, inactive compounds (NA) activities were taken to be the ones presenting $\mathrm{IC}_{50}=250 \mu \mathrm{mol} \mathrm{L}{ }^{-1}$. The dendrogram obtained (Figure 7) helps to justify the similarities between models 1 and 3 , and between models 2 and 4 . By also inserting the data concerning the NH3T3 cell line, it also appears that the mechanism of toxicity against normal cells probably has no relation to cytotoxic activity. As a basis for these results, it may be proposed that the cytotoxic effects of sub-sets 1 and 3 may occur by some similar mechanism between the two cell lines, and the same can be proposed for sub-sets 2 and 4 . Another information to be extracted from the dendrogram is that the similarity (approximately 0.54) between the sub-sets 1 and 3 is greater than that of sub-sets 2 and 4 (approximately 0.40 ), what can be confirmed by

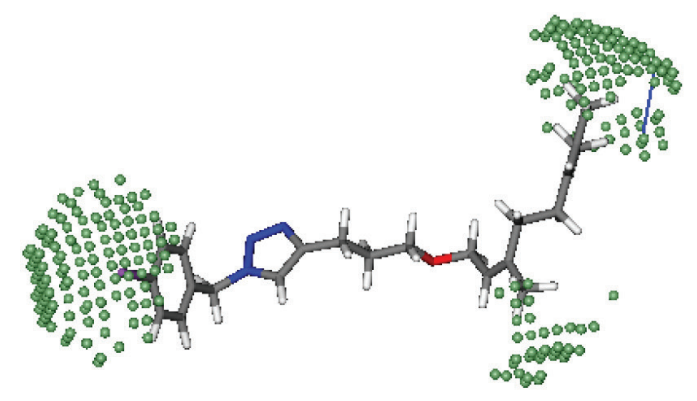

HL60 202_TIP-TIP

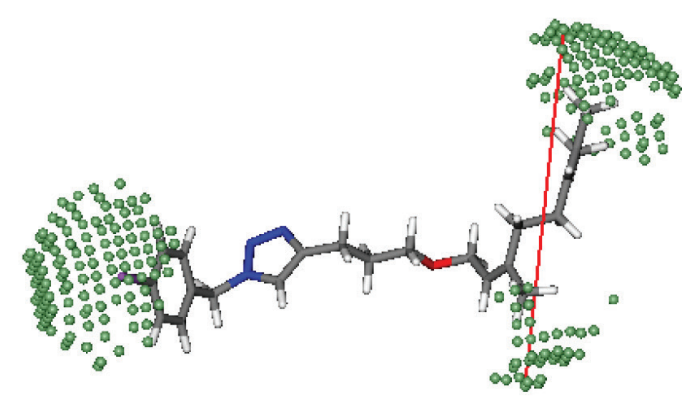

HL60 221_TIP-TIP calculating the Pearson's correlation coefficient, $r$, between cell lines activities from Table $1\left(\mathrm{r}_{\mathrm{HL} 60 / \mathrm{Jurkat}}=0.8\right.$ and $\mathrm{r}_{\text {Nalm6/B16F10 }}=0.7$ ).

These results may indicate that, from the structural modification of nerol, it would be possible to develop agents useful for the treatment of more than one type of cancer.

\section{Conclusions}

In summary, a series of nerol derivatives containing triazolic functionalities was designed and synthesized using the CuAAC (click reaction) as the key step. The compounds were evaluated against four different cancer cell lines types. The occurrence of a remarkable variability among the compounds suggests that the presence of various substituents in the aryl group in the triazolic portion, and their position as well, interfere with cell viability. In addition, the length of the aliphatic chain linking the nerol moiety to the triazolic portion also impacts the biological activity. The most cytotoxic compound 9f was also able to impair cell viability over time and migration. Overall, considering the biological activity results along with the calculated physicochemical properties, the triazolic nerol derivatives described herein seem favorable for further drug development procedures. The 3D-QSAR investigation points to the fact that two different mechanisms of action can be associated with the four types of cell lines used in the biological assays.

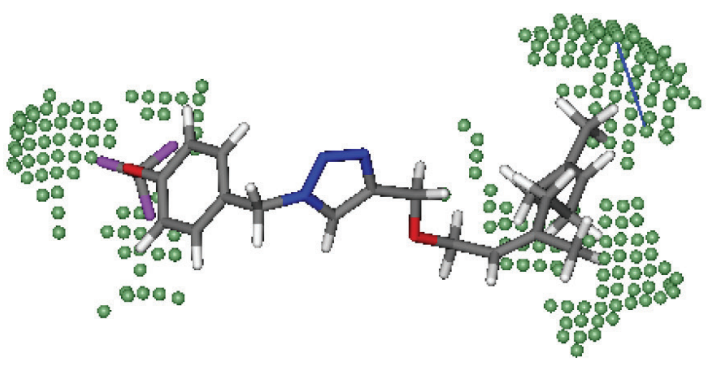

JURKAT 199_TIP-TIP

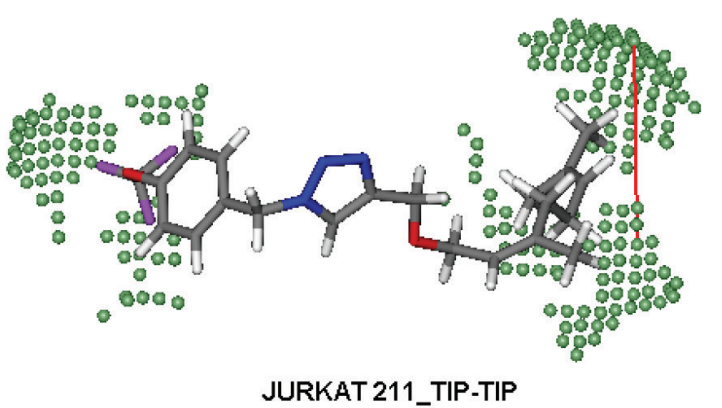

Figure 5. Graphical representations of similar GRIND descriptors in models 1 and 3 superimposed on the most active compounds of each subset. Blue line represents a descriptor with negative influence under activity and red line a descriptor with positive influence. Green spheres: TIP fields. 


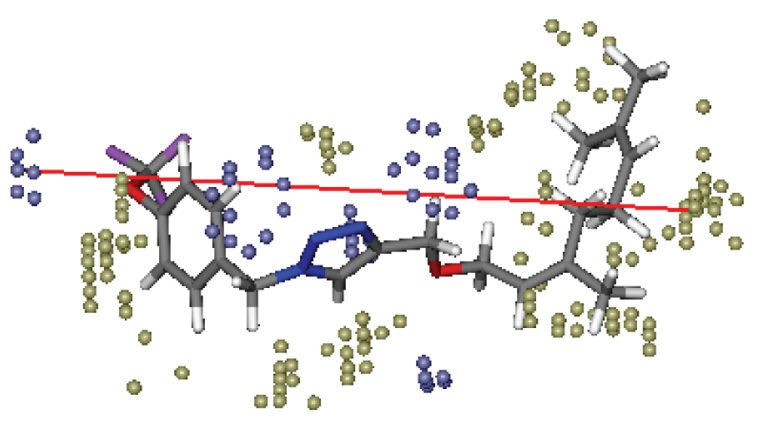

Nalm6 362_DRY-N1

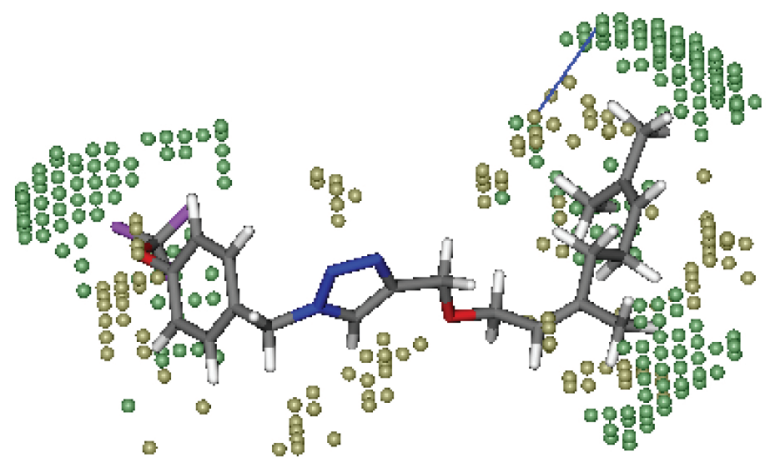

Nalm6 386_DRY-TIP
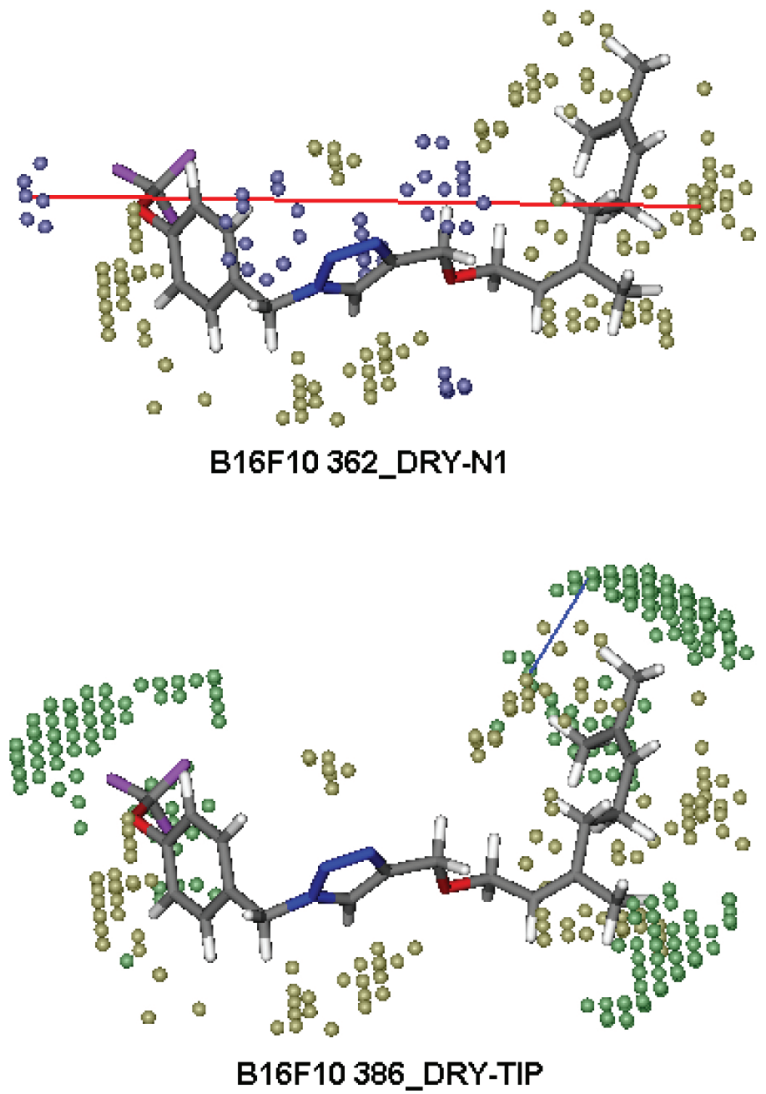

Figure 6. Graphical representations of similar GRIND descriptors in models 2 and 4 superimposed on the most active compounds of each subset. Blue line represents a descriptor with negative influence under activity, and red line a descriptor with positive influence. Green spheres: TIP fields; yellow spheres: DRY fields; blue spheres: N1 fields.

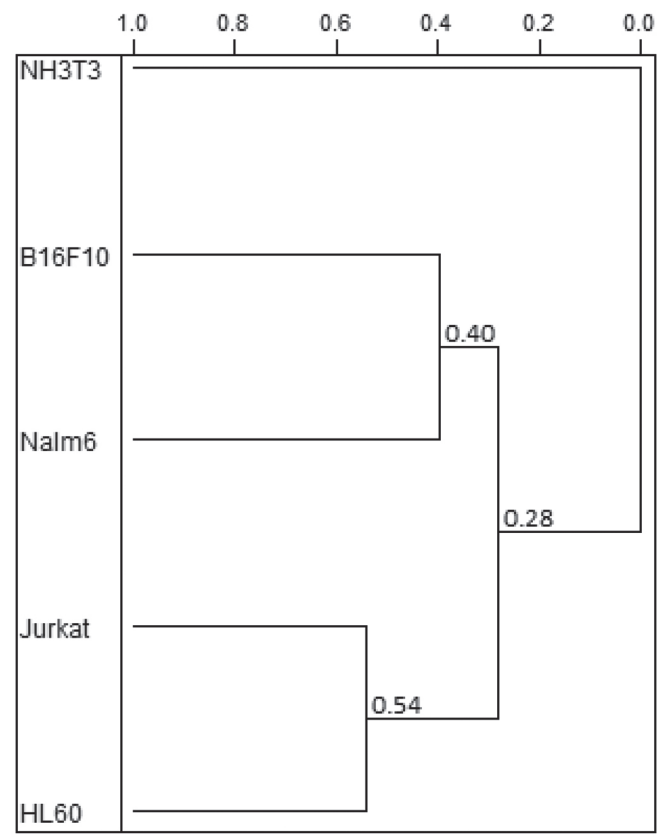

Figure 7. Dendrogram of biological activities (autoscaled data, flexible linkage) showing their similarity.

Taken the data described in the present investigation, it is assumed that nerol derivatives containing triazolic functionalities seem to be a scaffold to be explored toward the development of new agents against cancer.

\section{Supplementary Information}

Supplementary information (IR, NMR $\left({ }^{1} \mathrm{H}\right.$ and $\left.{ }^{13} \mathrm{C}\right)$ and MS spectra of the compounds, tables related to the QSAR study and the result of the effect compound $9 f$ on the human non-cancerous cell line HEK293) is available free of charge at http://jbcs.sbq.org.br as PDF file.

\section{Acknowledgments}

The authors are grateful to the following Brazilian agencies: Fundação de Amparo à Pesquisa de Minas Gerais (FAPEMIG), Fundação de Amparo à Pesquisa de São Paulo (FAPESP), Conselho Nacional de Desenvolvimento Científico e Tecnológico (CNPq), Coordenação de Aperfeiçoamento de Pessoal de Nível Superior (CAPES), Fundação de Amparo à Pesquisa do Rio Grande do Sul (FAPERGS), and Fundação Araucária (FAPPR) for financial support. Fundação Arthur Bernardes (FUNARBE) is also acknowledged for a fellowship to G. C. B. 


\section{References}

1. Butler, M. S.; J. Nat. Prod. 2004, 67, 2141.

2. Paterson, I.; Anderson, E. A.; Science 2005, 310, 451.

3. Koehn, F. E.; Carter, G. T.; Nat. Rev. Drug Discovery 2005, 4, 206.

4. Newman, D. J.; Cragg, G. M.; J. Nat. Prod. 2007, 70, 461.

5. Li, J. W. L.; Vederas, J. C.; Science 2009, 325, 161.

6. Harvery, A. L.; Edrada-Ebel, R.; Quinn, R. J.; Nat. Rev. Drug Discovery 2015, 14, 111.

7. Newman, D. J.; Cragg, M. G.; J. Nat. Prod. 2016, 79, 629.

8. Bhanot, A.; Sharma, R.; Noolvi, M. N.; Int. J. Phytomed. 2011, $3,09$.

9. Djilani, A.; Dicko, A.; Nutrition, Well-Being and Health; Bouayed, J., ed.; IntechOpen: India, 2012, ch. 7, p. 155-178.

10. Marques, T. H. C.; Marques, B. G. C. B.; Lima, D. S.; Siqueira, H. D. S.; Neto, J. D. N.; Branco, S. B. G.; Souza, A. A.; Souza, D. P.; Freitas, R. M.; World J. Neurosci. 2013, 3, 32.

11. Yamamoto, Y.; Hosokawa, M.; Kurihara, H.; Maoka, T.; Miyashita, K.; Bioorg. Med. Chem. Lett. 2008, 18, 4004.

12. Teixeira, R. R.; Bressan, G. C.; Pereira, W. L.; Ferreira, J. G.; Oliveira, F. M.; Thomaz, D. C.; Molecules 2013, 18, 1881.

13. Siqueira, R. P.; Barbosa, E. A. A.; Polêto, M. D.; Righetto, L. M.; Seraphim, T. V.; Salgado, R. L.; Ferreira, J. G.; Barros, M. V. A.; Oliveira, L. L.; Laranjeira, A. B. A.; Almeida, M. R.; Silva Júnior, A.; Fietto, J. L. R.; Kobarg, J.; Oliveira, E. B.; Teixeira, R. R.; Borges, J. C.; Yunes, J. A.; Bressan, G. C.; PLoS One 2015, 10, e0134882.

14. Silva, M. R.; Moreira, G. A.; Silva, R. A. G.; Barbosa, E. A. A.; Siqueira, R. P.; Teixeira, R. R.; Almeida, M. R.; Júnior, A. S. J.; Fietto, J. L. R.; Bressan, G. C.; Biomed. Res. Int. 2015, article ID 150514.

15. Maia, A. F. S.; Siqueira, R. P.; Oliveira, F. M.; Ferreira, J. G.; Silva, S. F.; Cauiby, C. A.; Oliveira, L. L.; Paula, S. O.; Souza, R. A. C.; Guilardi, S.; Bressan, G. C.; Teixeira, R. R.; Bioorg. Med. Chem. Lett. 2016, 26, 2810.

16. Lima, G. D. A.; Rodrigues, M. P.; Mendes, T. A. O.; Moreira, G. A.; Siqueira, R. P.; da Silva, A. M.; Vaz, B. G.; Fietto, J. L. R.; Bressan, G. C.; Machado-Neves, M.; Teixeira, R. R.; Toxicol. In Vitro 2018, 53, 1.

17. Kolb, H. C.; Sharpless, K. B.; Drug Discovery Today 2003, 8 , 1128.

18. Tron, G. C.; Pirali, T.; Billington, R. A.; Canonico, P. L.; Sorba, G.; Genazzani, A. A.; Med. Res. Rev. 2008, 28, 278.

19. Thirumurugan, P.; Matosiuk, D.; Jozwiak, K.; Chem. Rev. 2013, $113,4905$.

20. Kolb, H. C.; Finn, M. G.; Sharpless, K. B.; Angew. Chem., Int. Ed. 2001, 40, 2004.

21. Dheer, D.; Singh, V.; Shankar, R.; Bioorg. Chem. 2017, 71, 30.

22. Ma, N.; Wang, Y.; Zhao, B.-X.; Ye, W.-C.; Jiang, S.; Drug Des., Dev. Ther. 2015, 9, 1585.
23. Demchuk, D. V.; Samet, A. V.; Chernysheva, N. B.; Ushkarov, V. I.; Stashina, G. A.; Konyushkin, L. D.; Raihstat, M. M.; Firgang, S. I.; Philchenkov, A. A.; Zavelevich, M. P.; Kuiava, L. M.; Chekhun, V. F.; Blokhin, D. Y.; Kiselyov, A. S.; Semenova, M. N.; Semenov, V. V.; Bioorg. Med. Chem. 2014, 22, 738.

24. Praveena, K. S. S.; Durgadas, S.; Babu, N. S.; Akkenapally, S.; Kumar, C. G.; Deora, G. S.; Murthy, N. Y. S.; Mukkanti, K.; Pal, S.; Bioorg. Chem. 2014, 53, 8.

25. Khazir, J.; Hyder, I.; Gayatri, L.; Yandrati, L. P.; Nalla, N.; Chasoo, G.; Mahajan, A.; Saxena, A. K.; Alam, M. S.; Qazi, G. N.; Kumar, H. M. S.; Eur. J. Med. Chem. 2014, 82, 255.

26. Allam, M.; Bhavani, A. K. D.; Mudiraj, A.; Ranjan, N.; Thipanna, M.; Babu, P. P.; Eur. J. Med. Chem. 2018, 156, 43.

27. Tsai, Y.; Etichetti, C. M. B.; Di Benedetto, C.; Girardini, J. E.; Martins, F. T.; Spanevello, R. A.; Suárez, A. G.; Sarotti, A. M.; J. Org. Chem. 2018, 83, 3516.

28. Hou, W.; Luo, Z.; Zhang, G.; Cao, D.; Li, D.; Ruan, H.; Ruan, B. H.; Su, L.; Xu, H.; Eur. J. Med. Chem. 2017, 138, 1042.

29. Borgati, T. F.; Alves, R. B.; Teixeira, R. R.; Freitas, R. P.; Perdigão, T. G.; Silva, S. F.; Santos, A. A.; Bastidas, A. J. O.; J. Braz. Chem. Soc. 2013, 24, 953.

30. Siqueira, R. P.; Barros, M. V. A.; Barbosa, E. A. A.; Onofre, T. S.; Gonçalves, V. H. S.; Pereira, H. S.; Júnior, A. S.; Oliveira, L. L.; Oliveira, M. R.; Fietto, J. L. R.; Teixeira, R. R.; Bressan, G. C.; Eur. J. Med. Chem. 2017, 134, 97.

31. Rasband, W. S.; ImageJ, version 1.8.0; U. S. National Institutes of Health, Bethesda, Maryland, USA, 1997-2018. Available at https://imagej.nih.gov/ij/, accessed in October 2018.

32. GraphPad Prism, version 5.00 for Windows; GraphPad Software, Inc., La Jolla California, USA. Available at www. graphpad.com, accessed in October 2018.

33. MarvinSketch, version 15.4.6.0; ChemAxon, Budapest, Hungary, 2015.

34. LigPrep, version 3.6; Schrödinger, LLC, New York, NY, USA, 2015.

35. QikProp, version 4.6; Schrödinger, LLC, New York, NY, USA, 2015.

36. Irwin, J. J.; Sterling, T.; Mysinger, M. M.; Bolstad, E. S.; Coleman, R. G.; J. Chem. Inf. Model. 2012, 52, 1757.

37. Frisch, M. J.; Trucks, G. W.; Schlegel, H. B.; Scuseria, G. E.; Robb, M. A.; Cheeseman, J. R.; Scalmani, G.; Barone, V.; Petersson, G. A.; Nakatsuji, H.; Li, X.; Caricato, M.; Marenich, A.; Bloino, J.; Janesko, B. G.; Gomperts, R.; Mennucci, B.; Hratchian, H. P.; Ortiz, J. V.; Izmaylov, A. F.; Sonnenberg, J. L.; Williams-Young, D.; Ding, F.; Lipparini, F.; Egidi, F.; Goings, J.; Peng, B.; Petrone, A.; Henderson, T.; Ranasinghe, D.; Zakrzewski, V. G.; Gao, J.; Rega, N.; Zheng, G.; Liang, W.; Hada, M.; Ehara, M.; Toyota, K.; Fukuda, R.; Hasegawa, J.; Ishida, M.; Nakajima, T.; Honda, Y.; Kitao, O.; Nakai, H.; Vreven, T.; Throssell, K.; Montgomery Jr., J. A.; Peralta, A. 
E.; Ogliaro, F.; Bearpark, M.; Heyd, J. J.; Brothers, E.; Kudin, K. N.; Staroverov, V. N.; Keith, T.; Kobayashi, R.; Normand, J.; Raghavachari, K.; Rendell, A.; Burant, J. C.; Iyengar, J.; Tomasi, S. S.; Cossi, M.; Millam, J. M.; Klene, M.; Adamo, C.; Cammi, R.; Ochterski, J. W.; Martin, R. L.; Morokuma, K.; Farkas, O.; Foresman, J. B.; Fox, D. J.; Gaussian 09; Gaussian, Inc., Wallingford, 2009.

38. Molfetta, F. A.; Bruni, A. T.; Rosseli, F. P.; Silva, A. B. F.; Struct. Chem. 2007, 18, 49.

39. Kawasuji, T.; Fuji, M.; Yoshinaga, T.; Sato, A.; Fujiwara, T.; Kiyama, R.; Bioorg. Med. Chem. 2006, 14, 8420.

40. Weigend, F.; Ahlrichs, R.; Phys. Chem. Chem. Phys. 2005, 7 , 3297.

41. Pentacle, version 1.05; Molecular Discovery Ltd., Barcelona, Spain, 2010.

42. Pastor, M.; Cruciani, G.; McLay, I.; Pickett, S.; Clementi, S.; J. Med. Chem. 2000, 43, 3233.

43. Teófilo, R. F.; Martins, J. P.; Ferreira, M. M. C.; J. Chemom. 2009, 23, 32.

44. Martins, J. P. A.; Ferreira, M. M. C.; Quim. Nova 2013, 36, 554.

45. Kiralj, R.; Ferreira, M. M. C.; J. Braz. Chem. Soc. 2009, 20, 770 .
46. Eriksson, L.; Jaworska, J.; Worth, A. P.; Cronin, M. T.; McDowell, R. M.; Gramatica, P.; Environ. Health Perspect. 2003, $111,1361$.

47. Pirouette, version 4; Infometrix Inc., Woodinville, WA, USA, 2003.

48. Gazolla, P. A. R.; Teixeira, R. R.; da Silva, A. M.; Vaz, B. G.; Vasconcelos, G. A.; Siqueira, R. P.; Gonçalves, V. H. S.; Pereira, H. S.; Bressan, G. C.; Quim. Nova 2018, 41, 497.

49. Tatman, D.; Mo, H.; Cancer Lett. 2002, 175, 129.

50. Carnesecchi, S.; Schneider, Y.; Ceraline, J.; Duranton, B.; Gosse, F.; Seiler, N.; Raul, F.; J. Pharmacol. Exp. Ther. 2001, 298, 197.

51. Taga, T.; Shimomura, Y.; Horikoshi, Y.; Ogawa, A.; Itoh, M.; Okada, M.; Ueyama, J.; Higa, T.; Watanabe, A.; Kanegane, H.; Iwai, A.; Saiwakawa, Y.; Kogawa, K.; Yamanaka, J.; Tsurusawa, M.; Pediatr. Blood Cancer 2011, 57, 36.

52. Lipinski, C. A.; J. Pharm. Toxicol. Methods 2000, 44, 235.

53. Veber, D. F.; Johnson, S. R.; Cheng, H. Y.; Smith, B. R.; Ward, W.; Kopple, K. D.; J. Med. Chem. 2002, 45, 2615.

Submitted: August 2, 2018 Published online: October 10, 2018 